\title{
Close shock detection using time-frequency Prony modeling
}

\author{
Nadine Martin, Pierre Jaussaud and François Combet
}

\section{Laboratoire des Images et des Signaux} (INPG-CNRS)

BP46, 38402 Saint Martin d'Hères Cedex France

nadine.martin@lis.inpg.fr 


\begin{abstract}
In many cases, modeling a mechanical process may require a good understanding of signals issued from the system, as vibration accelerations. This is particularly the case when shocks are responsible of the vibrations. In the case of critical systems, each shock induces natural modes excitation with damped sines amplitudes. Identification of the shocks (occuring instants and induced vibrations) is a very important step of the analysis. However, when successive shocks are very close, their separation and their individual identification are not easy. In that paper, we adapt the well-known stationary Prony model to this non stationary context. We propose a method where shocks instants detection and parameters shocks estimation are separated. We illustrate the performances of the method on COR curves. In a last part, we apply it to a real acceleration signal recorded on a chairlift running over a compression tower in a rope transport plant where 48 shocks are expected, some of them being separated by only a few milliseconds.
\end{abstract}




\section{Introduction}

The aim of this paper is to study the response of a mechanical system to a succession of shocks. It deals with linear systems which conditions are such that their responses to shocks are damped sines. More generally, this paper also concerns all transitory signals which wave shape can have a model defined by a damped sine. We introduce an excitation model, which stems from mechanics. This model represents successive shocks occurring at different instants, not necessarily regularly distributed, with a shock model defined by a Dirac function. We call this model a multi-shocks model. The objective of that paper is to propose a method for modeling the output signal of systems excited this way.

We first illustrate the fact that the Fourier analysis rapidly reaches its limits even by a time frequency approach with a short time Fourier transform. Blind deconvolution could be applied to the output signals for estimating the input excitation without a priori knowledge on the system response. These inverse methods are tackled with order 2 , by minimization of a prediction error power, with high order statistics [LAC 97] or, more recently, with mutual information, by adapting methods from source separation [TAL 01]. These methods assume stationary system parameters. These approaches are not considered in that paper and the reader can refer to [COM 02] for an application to multi-shock signals.

In this paper, we choose an approach, which yields a model of the response and which takes into account the nonstationary aspect of the problem. In signal processing literature, a damped sine exactly refers to the Prony model. This model was introduced in a stationary context of fluid dynamics [PRO 95]. We propose to extend this model to a nonstationary context as the one mentioned above. The system response can change with time eventually. When oscillations are weakly damped, a model can be identified on a gliding window, such as for instance the biomedical application presented in [GAR 00]. But, if damping must be taken into account, an appropriate processing must be used. In 1982, Atal [ATA 82] proposed to iterate a deflation algorithm: estimating of the first pulse, then subtracting it from the signal, estimating of the next pulse, etc. In 1996, for analyzing electromagnetic transitory signals, S. Yvetot [YVE 96] presented an exhaustive study that introduces a multi-pulse model based on the Prony approach where pulses instant detection were performed by a wavelet transform.

The method we propose here has some common points with that developed by Yvetot. We apply the Prony method on a gliding window. The only constraint is a local stationarity requirement, which is reasonable to assume with shocks. Window width is an important parameter, which must be equal to the system response time. We propose to estimate this 
width by using the Prony estimator properties on variable window width. Using Prony method for each shock requires a first step to detect the instant at which shock occurs. The originality of our approach is to propose an initial step for instant detection, also based on Prony method. It is linked to Prony sensitivity to non-stationary signals and namely to phase advance or delay.

In Section 2, we derive the multi-shock model from a mechanical point of view. In Section 3, we present a rope chairlift compression tower, excited by such a model. In Section 4, we comment and illustrate the limits of the Fourier analysis in that context. Section 5 briefly recalls the stationary Prony method and is then focused on the correlation Prony one. We also investigate a crucial problem of all parametric estimators, which needs inverse matrices computing. All methods are very sensitive to the matrices conditioning. We propose an original and simple test for avoiding a bad conditioning. In Section 6, we derive the method we propose, a detection/estimation based on time-frequency Prony. It will reveal to be particularly efficient when shocks are close to each other and when the response to each shock is very short, of about one oscillation period. Performances are illustrated by mean of ROC curves. Knowledge of the mechanical structure response to shocks is also required to calculate the lifetime of the structure [CEC 87]. In Section 7 , this method is applied to an acceleration signal occurring at hanging top of the chairlift running under a compression tower. It allows us to model the acceleration that is induced in this type of situation, thus increasing the possibilities of managing safety problems in chairlifts.

\section{2. «Multi-shock » model}

A shock occurs when a mechanical system is submitted to a rapid variation of its speed (or momentum $\vec{P}=M \vec{V}$ ), either in terms of amplitude or direction. Through the basic mechanics equation $\vec{F}=\frac{d \vec{P}}{d t}$, this shock can be assimilated into a force applied during a short time. It is well known that a shock on a structure will excite its resonance modes. Let us consider a discrete linear system with $P$ degrees of freedom and losses proportional to speed. It is thus ruled by a linear second order differential system with constant coefficients. If the shock or excitation input is defined as the $P$ dimension vector $\mathbf{e}(t)$, the response movement (strain or deformation) amplitude is the physical displacement of the system from its equilibrium position for each degree of freedom. This response defined as a $\mathrm{P}$ dimension vector $\mathbf{y}(t)$ is solution of [BRU 55]

$$
\mathbf{M} \ddot{\mathbf{y}}(t)+\mathbf{C} \dot{\mathbf{y}}(t)+\mathbf{K} \mathbf{y}(t)=\mathbf{e}(t)
$$


where $\mathbf{e}(t)$ defines the excitation or input of the system, measured as an acceleration, $\dot{\mathbf{y}}(t)$ and $\ddot{\mathbf{y}}(t)$ are speed and acceleration of the displacement, $\mathbf{M}$ the $P \times P$ inertia matrix, $\mathbf{C}$ the $P \times P$ damping matrix and $\mathbf{K}$ the $P \times P$ stiffness matrix. Setting $\mathbf{w}(t)=\left[\begin{array}{c}\mathbf{y}(t) \\ \dot{\mathbf{y}}(t)\end{array}\right]$, the order of the system (1) is reduced by 1 and can be written as

$$
\dot{\mathbf{w}}(t)=\mathbf{B} \mathbf{w}(t)+\left[\begin{array}{c}
0 \\
\mathbf{M}^{-1} \mathbf{e}(t)
\end{array}\right]
$$

where the $2 P \times 2 P$ matrix $\mathbf{B}$ is defined as $\mathbf{B}=\left(\begin{array}{cc}\mathbf{0} & \mathbf{I} \\ -\mathbf{M}^{-1} \mathbf{K} & -\mathbf{M}^{-1} \mathbf{C}\end{array}\right)$ with $2 P$ complex conjugate eigenvalues $\lambda_{p}$ in such way as to have [NEW 89]

$$
\mathbf{V}_{2 P}^{T} \mathbf{B} \mathbf{V}_{2 P}=\left[\operatorname{diag}\left(\lambda_{1} \ldots \lambda_{2 P}\right)\right]
$$

where $\left[\operatorname{diag}\left(\lambda_{1} \ldots \lambda_{2 P}\right)\right]$ is a $2 P \times 2 P$ diagonal matrix of term $\lambda_{p} . \mathbf{V}_{2 P}$ is the $2 P \times 2 P$ orthogonal matrix whose columns are eigenvectors of $\mathbf{B}$ and represents the relative amplitude of the movement displacement in relation to each mode for the $P$ first values and the relative velocity in relation to each mode for the $P$ next values. So we can write

$$
\mathbf{V}_{2 P}=\left[\begin{array}{c}
\mathbf{V}_{u p} \\
\mathbf{V}_{\text {down }}
\end{array}\right] \text { and } \mathbf{V}_{2 P}^{-1}=\left[\begin{array}{ll}
\mathbf{V}_{\text {left }}^{-1} & \mathbf{V}_{\text {right }}^{-1}
\end{array}\right]
$$

with $\mathbf{V}_{u p}$ the $P \times 2 P$ orthogonal matrix relative to the displacement and $\mathbf{V}_{\text {down }}$ the $P \times 2 P$ orthogonal matrix relative to the velocity, $\mathbf{V}_{\text {left }}^{-1}$ and $\mathbf{V}_{\text {right }}^{-1}$ are $2 P \times P$ matrices. The solution of Eq. (1) is the sum of two terms

$$
\mathbf{y}(t)=\mathbf{y}_{F}(t)+\mathbf{y}_{L}(t)
$$

The $P$ dimension vector $\mathbf{y}_{F}(t)$ is the forced contribution due to excitation $\mathbf{e}(t)$ whereas the $P$ dimension vector $\mathbf{y}_{L}(t)$ represents the free oscillations created by the shock. Mathematically speaking, $\mathbf{y}_{F}(t)$ is a particular solution of the full equation and $\mathbf{y}_{L}(t)$ is a general solution of the system without its right-handed term, the homogeneous system [BAS 68].

\subsection{Expression of the forced solution}

A particular solution $\mathbf{y}_{F}(t)$ of system (1) can be given as [NEW 89] 


$$
\mathbf{y}_{F}(t)=\mathbf{V}_{u p}\left[\operatorname{diag}\left(e^{\lambda_{1} t} \ldots e^{\lambda_{2} p^{t}}\right)\right] \int_{0}^{t}\left[\operatorname{diag}\left(e^{-\lambda_{1} s} \ldots e^{-\lambda_{2} P}\right)\right] \mathbf{V}_{r i g h t}^{-1} \mathbf{M}^{-1} \mathbf{e}(s) d s
$$

with $\left[\operatorname{diag}\left(e^{\lambda_{1} t} \ldots e^{\lambda_{2} P^{t}}\right)\right]$ a $2 P \times 2 P$ diagonal matrix of term $e^{\lambda_{i} t}$. Each component of $\mathbf{y}_{F}(t)$ is the sum of $P$ convolution products between each component of the excitation vector and a term of a $P \times P$ matrix. This matrix is the system impulse response $\mathbf{h}(t)$. Eq. (6) can be written as

$$
\mathbf{y}_{F}(t)=\int_{-\infty}^{+\infty} \mathbf{h}(t-s) \mathbf{e}(s) d s
$$

where $\mathbf{h}(t)$ is defined as

$$
\mathbf{h}(t)=u(t) \mathbf{V}_{u p}\left[\operatorname{diag}\left(e^{\lambda_{1} t} \ldots e^{\lambda_{2} t}\right)\right] \mathbf{V}_{\text {right }}^{-1} \mathbf{M}^{-1}
$$

where $u(t)$ is Heavyside function, which is zero when $t<0$ and 1 when $t>0$. Given that we consider the system input as a force and the system output as displacement, the impulse response has to be homogeneous to $s . \mathrm{kg}^{-1}$, as it is.

\subsection{Expression of the free solution}

For a linear system where the response is equal to the sum of individual responses of each eigenmode, the free solution $\mathbf{y}_{L}(t)$ of system (1) can be written as

$$
\mathbf{y}_{L}(t)=\sum_{p=1}^{2 P} u(t) c_{p} e^{\lambda_{p} t} \mathbf{v}_{u p_{p}}
$$

The $P$ dimension vector $\mathbf{v}_{u p_{p}}$, defined by the $p^{\text {th }}$ column of matrix $\mathbf{V}_{u p}$, represents the relative amplitudes of the movement of each mass for the mode $(p)$. The constants $c_{p}$, with unit $m$, are determined from the initial conditions that the system displacement $\mathbf{y}(t)$ must satisfy.

\subsection{Model of the excitation}

Let us now consider a sequence of Dirac shocks occurring at instants $t_{m}$. The so-defined input $\mathbf{e}_{M}(t)$ can be written as

$$
\mathbf{e}_{M}(t)=\sum_{m=1}^{M} \delta\left(t-t_{m}\right) \mathbf{e}_{m}
$$


where the $P$ dimension vector $\mathbf{e}_{m}$ stands for amplitude of the $\mathrm{m}^{\text {th }}$ shock and is homogeneous to a force. $\delta(t)$ stands for Dirac function and $M$ is the shock number. This excitation model is very simple. Others can be written with more complex functions than Dirac one, such as finite duration pulses or serrated functions. Examples are given in [LAL 99].

At instant $t_{m}$, the response $\mathbf{y}_{m}(t)$ to a single excitation $\delta\left(t-t_{m}\right) \mathbf{e}_{m}$ is drawn from Eq. (5) with the forced solution given by Eq. (7) and the free solution by Eq. (9). So, $\mathbf{y}_{m}(t)$ can be written as

$$
\mathbf{y}_{m}(t)=\mathfrak{I} \mathbf{h}\left(t-t_{m}\right) \mathbf{e}_{m}+\sum_{p=1}^{2 P} u\left(t-t_{m}\right) c_{m_{p}} e^{\lambda_{p}\left(t-t_{m}\right)} \mathbf{v}_{u p_{p}}
$$

where $\mathfrak{I}=\int_{-\infty}^{+\infty} \delta(s) d s=1$ with unit $s$, [LAL 99].

Constants $c_{m_{p}}$ are now indexed by $m$ to refer it to the instant $t_{m}$. Theses constants are such that $\mathbf{y}_{m}(t)$ satisfies the initial conditions of the system. These conditions are defined at each instant $t_{m}$ from the state of the system given by all preceding shocks. Thus, initial conditions at instant $t_{m}$ are defined by the initial position $\mathbf{y}_{\mathrm{ot}_{\mathrm{m}}}=\mathbf{y}_{m-1}\left(t_{m}\right)$ and by the initial speed $\dot{\mathbf{y}}_{m-1}\left(t_{m}\right)$.

Response $\mathbf{y}(t)$ to the $M$ excitations defined by Eq. (10) is the sum of the M responses $\mathbf{y}_{m}(t)$ defined in Eq. (11). Taking Eq. (8) into account, $\mathbf{y}(t)$ can be written as

$$
\mathbf{y}(t)=\sum_{m=1}^{M} u\left(t-t_{m}\right)\left(\mathfrak{I} \mathbf{V}_{u p}\left[\operatorname{diag}\left(e^{\lambda_{1}\left(t-t_{m}\right)} \ldots e^{\lambda_{2 P}\left(t-t_{m}\right)}\right)\right] \mathbf{V}_{r i g h t}^{-1} \mathbf{M}^{-1} \mathbf{e}_{m}+\sum_{p=1}^{2 P} c_{m_{p}} e^{\lambda_{p}\left(t-t_{m}\right)} \mathbf{v}_{u p_{p}}\right)
$$

Given that $\lambda_{p}=-\alpha_{p}+j \Omega_{p}$, each coordinate $y_{i}(t)$ of vector $\mathbf{y}(t)$ has the form

$$
y_{i}(t)=\sum_{m=1}^{M} \hat{y}_{m i}\left(t-t_{m}\right) u\left(t-t_{m}\right)
$$

where

$$
\hat{y}_{m i}(t)=\sum_{p=1}^{2 P} a_{m i p} e^{-\alpha_{p} t} e^{j \Omega_{p} t}
$$

with the complex amplitude $a_{\text {mip }}$ defined by 


$$
a_{m i p}=\mathfrak{I}\left(\sum_{j=1}^{P} w_{p j} e_{m_{j}}\right) v_{u p_{i p}}+c_{m_{p}} v_{u p_{i p}}
$$

where $\mathbf{V}_{r i g h t}^{-1} \mathbf{M}^{-1}=\left[w_{p j}\right]$ with unit s.kg ${ }^{-1}$ and $\mathbf{V}_{u p}=\left[v_{u p_{i p}}\right]$ dimensionless.

The complex amplitudes $a_{\text {mip }}$ and real parameters $\alpha_{p}, \Omega_{p}$ and $t_{m}$ completely characterise the system response to excitation $\mathbf{e}_{M}(t)$. Response (13) will henceforth be called multi-shock model. If the shocks occur before the vibrations of the former shock disappeared, the interpretation of observations is difficult, either in time or in frequency domain. Our aim is to characterise each response to an elementary shock, even when these are close to each other, that is to say when $\left(t_{m+1}-t_{m}\right)$ is about $\min _{p=1, P}\left(2 \pi / \Omega_{p}\right)$.

From an experimental point of view, the signal that is measured is very often an acceleration. In this case, the measured signal must be compared to the second order derivative of $y_{i}(t)$ that we can write as

$$
\ddot{y}_{i}(t)=\sum_{m=1}^{P} \sum_{p=1}^{2 P} a_{m i p}\left[\left(\Omega_{p}^{2}-\alpha_{p}^{2}\right)^{2}+4 \alpha_{p}^{2} \Omega_{p}^{2}\right]^{1 / 2} e^{-\alpha_{p}\left(t-t_{m}\right)} e^{j\left(\Omega_{p}\left(t-t_{m}\right)+\operatorname{arctg}\left(\frac{2 \alpha_{p} \Omega_{p}}{\Omega_{p}^{2}-\alpha_{p}^{2}}\right)\right)} u\left(t-t_{m}\right)
$$

The only difference between Eqs. (14) and (16) is an amplitude factor and an additive phase parameter function of the system parameters. In both cases, the response can be written under the form of a damped sine sum.

\section{Rope chairlift compression tower and shocks}

In the vertical plane of a rope chairlift compression tower, a rocker with two wheels has only one degree of freedom as the direction of the line joining the wheels' center is the only required parameter to describe its behaviour. In a twelve wheel rocker, the rocker position can be described by 11 angles, thus leading to 11 degrees of freedom: 6 for the 6 two wheel rockers, 2 for the 2 four-wheel rockers, 2 for the 2 six-wheel rockers and one for the single 12 wheel rocker. Fig. 1c shows the links corresponding to each degree of freedom (1 color by link).

This analysis supposes that the wheels are independent and that the rope is elastic. This is not the case, first due to the high rope rigidity (raised by rope tension), and second due to the rope/wheel contacts. To analyze that point, we calculated the shape of a 4-wheel rocker when the first wheel is shifted away from the rope (see Fig. 2a). In this example, the shift follows a sine law one period along. It can be observed that $T E 3$, the link angle for wheels 3-4 (see Fig. 2b), is constant and that the angle for global rocker $T E 1$, is just one fourth of the excited beam angle $T E 2$, the 
link angle for wheels $1-2$. For 8 wheels, this ratio would be $1 / 8$ and so on. This result could justify an approximation of the degree of freedom of a 12-wheel rocker to 1 .

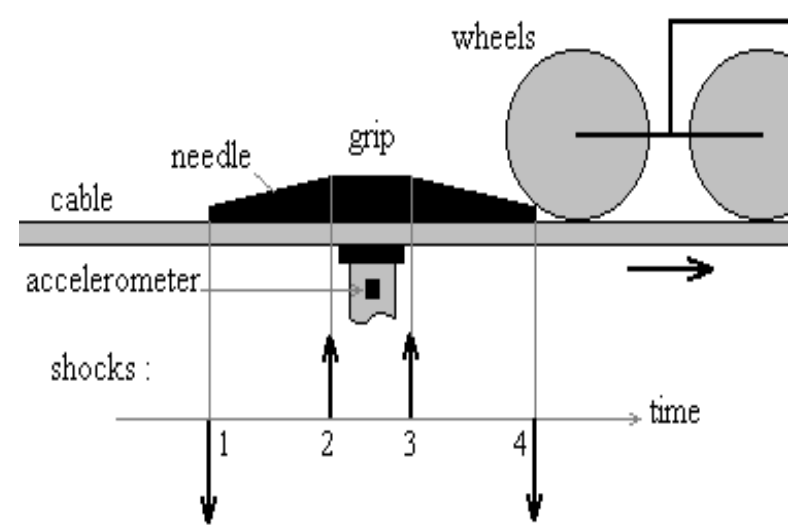

$1 \& 4$ : needles' shocks ; 2 \& 3 : grip's flat shocks

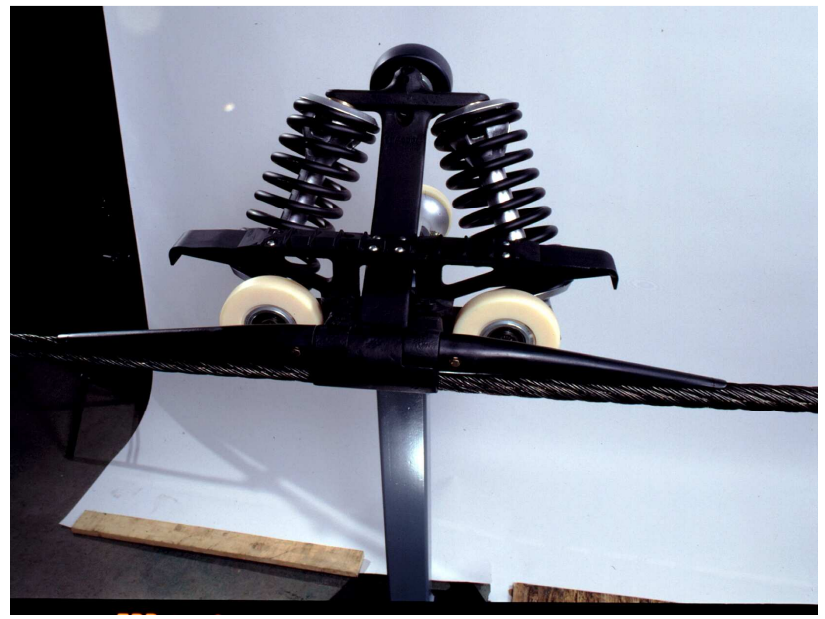

b - A detachable grip (by courtesy of POMA S.A.)

- ROTATION AXIS

RIGID LINK

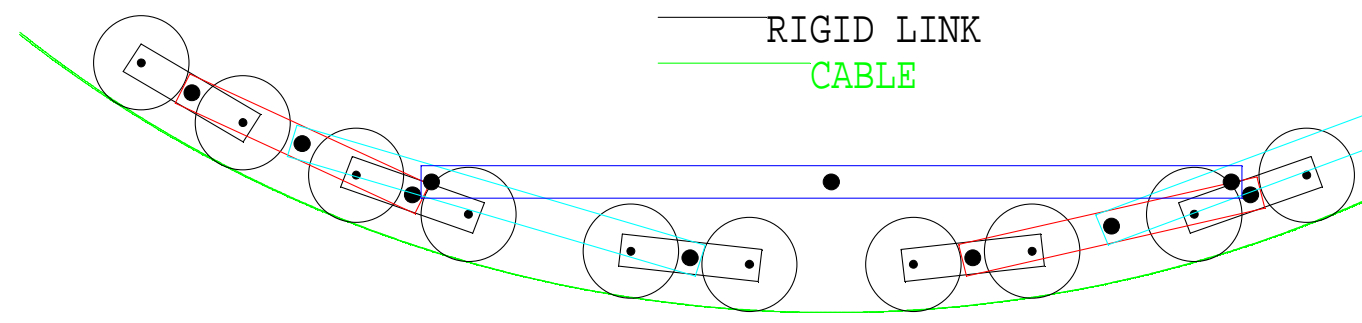

c- Wheels distribution on a rocker

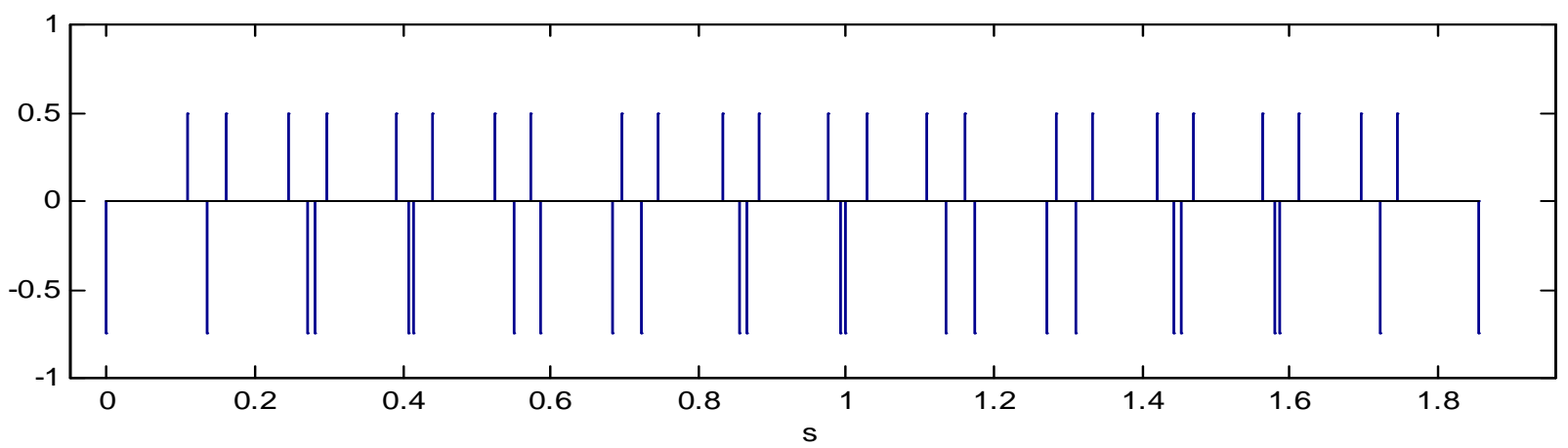

d- Rope chairlift excitation: shock instant distribution

Fig. 1. Presentation of the rocker model to be analysed at speed $4,16 \mathrm{~m} / \mathrm{s}$.

In fact, the system is more complex. The complete system includes a 12-wheel rocker, and also the rope and the tower. The last one could have flexions. Furthermore, the sensor is on the top of the chair suspension. The 12-wheel rocker is governed by equation system (1), where the displacement vector $\mathbf{y}(t)$ represents link angles between wheels. The degree of freedom is theoretically 11 but one angle seems playing a leading part. The sensor measures the vertical 
component of the acceleration. Under the assumption of small angles, this measure is equal to a linear combination of each component $y_{i}(t)$ described by Eq. (16).

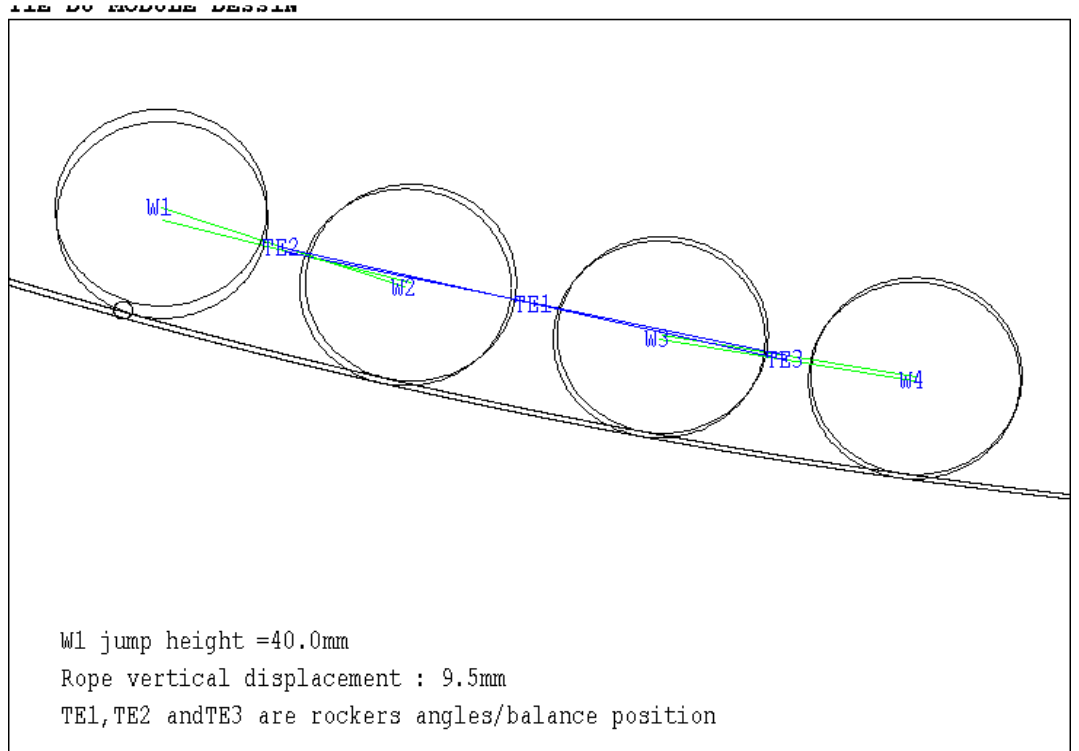

a-Two extreme positions in a four-wheel rocker when the first wheel is shifted away from the rope in the rocker plane.

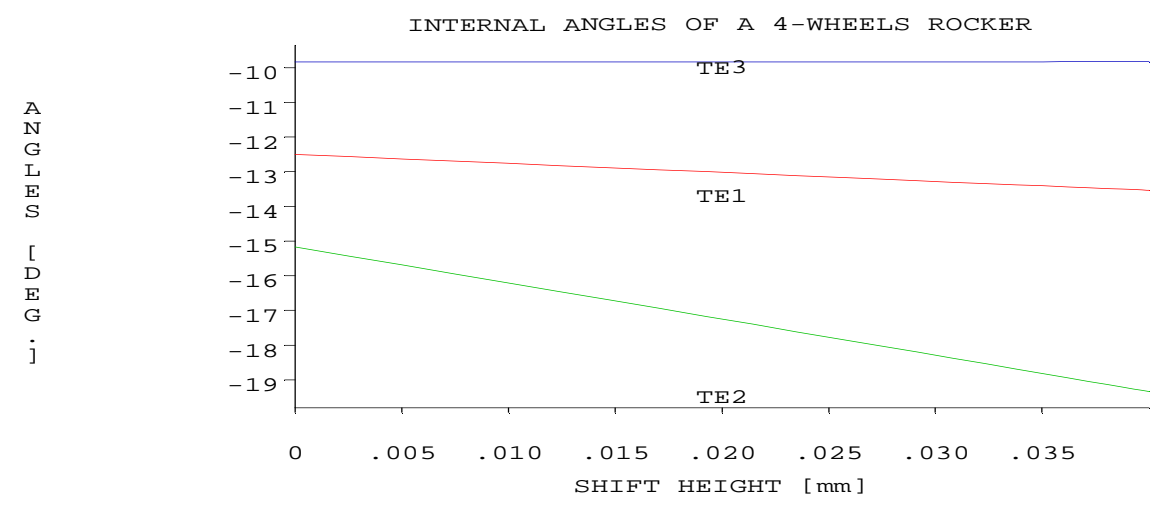

b-Sub-rocker angular movement in a four-wheel rocker when the first wheel is shifted away from the rope in the rocker plane

Fig. 2. Study of a four-wheel rocker

What about the excitation? In a rope chairlift compression tower, the rope goes under the rocker wheels. In this case, the grip upper part must insert between the rope and the wheels. At each grip angle, the speed vector direction changes being always vertical as shown in Fig. 1a. This occurs 4 times for each wheel. For instance, for a 12-wheel rocker, 48 shocks occur. The 2 grip ends correspond to a vertical downward speed variation of the grip and the two middle angles correspond to a vertical upward variation with smaller amplitude. Furthermore, shock intervals highly depend on grip and rocker geometry and are not regularly distributed. Using the wheel diameter and the rope speed, we can find when the grip/wheel shocks will occur and draw a scheme of these shocks. Grip presented in Fig. 1b running under the rocker 
of Fig. 1c gives a shock distribution presented in Fig. 1d in the case of a speed of $4.16 \mathrm{~m} / \mathrm{s}$. We assume that the impulse response is the same whatever the shock position is. The shock instant calculation takes into account the fact that the end shocks do not occur when the grip end goes under the wheel center but slightly before or after this point (see Fig. 1a).

At this time, we are not able to derive the equation system for such a mechanical system and such an excitation. In this paper, we propose a signal processing approach, which could help to model such a complex system. The method we propose is supported by the fact that Eq. (16) is a sum of damped sines, where damping factors and pulsations are those of the eigenmodes. The model does not take account of system parts, which act on the amplitudes and on the phases only.

\section{Fourier analysis of a response to multi-shocks signal}

Fourier analysis is a classic approach for physicists. Fourier transform $Y_{i}(\omega)$ of the response (13) can be written as

$$
Y_{i}(\omega)=\sum_{m=1}^{M} \sum_{i=1}^{2 P} \frac{a_{m i p}}{\alpha_{p}+j\left(\omega-\Omega_{p}\right)} e^{-j \omega t_{m}}
$$

The difficulty of this method is that it only gives global information on the spectrum. As several shocks occur, an extra phase term is introduced for each term of the sum. If shocks are regularly spaced and if the structure mode number is weak, it is possible to work with Eq. (17). Used together with spectrum interpretation technique and harmonics detection [DUR 99], [MAR 02], the method can lead to a good characterisation of the system with a good frequency resolution depending on the time extension of the signal. Nevertheless, in a more general case, using Fourier analysis turns out to be less fruitful. Let us come back to the chairlift acceleration signal described in Fig. 1. The experimental signal can be observed in Fig. 3 together with its global frequency analysis. The sampling frequency is $300 \mathrm{~Hz}$, which is enough given that the frequencies excited by the system are lower than $80 \mathrm{~Hz}$. This sampling rate was validated by other experiments using higher sampling frequency such as $1000 \mathrm{~Hz}$. As seen in Section 3, the 12-wheel rocker has theoretically 11 vibration modes. Global Fourier analysis failed in extracting the shock parameters. It might be interesting to use a narrow time-gliding window. This will provide time evolution of the frequency content of the signal. A spectrogram of the recorded signal of Fig. 3 is plotted in Fig. 4 with Blackman window of length 214 ms (64 points). We can see this method is however not very efficient, as its frequency resolution depends on the window size. This should be very small if we intend to separate closed shocks. In the case of the above rocker and grip, shock separation time is between $3 \mathrm{~ms}$ and $70 \mathrm{~ms}$ with a mean separation equal to $27 \mathrm{~ms}$. A spectrogram with 64 points 
window is not able to separate the different shocks in time. A smaller window length has an unacceptable frequency resolution. The limits of a Fourier based approached are thus rapidly reached. The mean shock separation is around one period of the eigenmode, which is estimated to $33 \mathrm{~ms}$ (see Section 7). In that context, only parametric method, which assumes a data model, can reach such a performance.
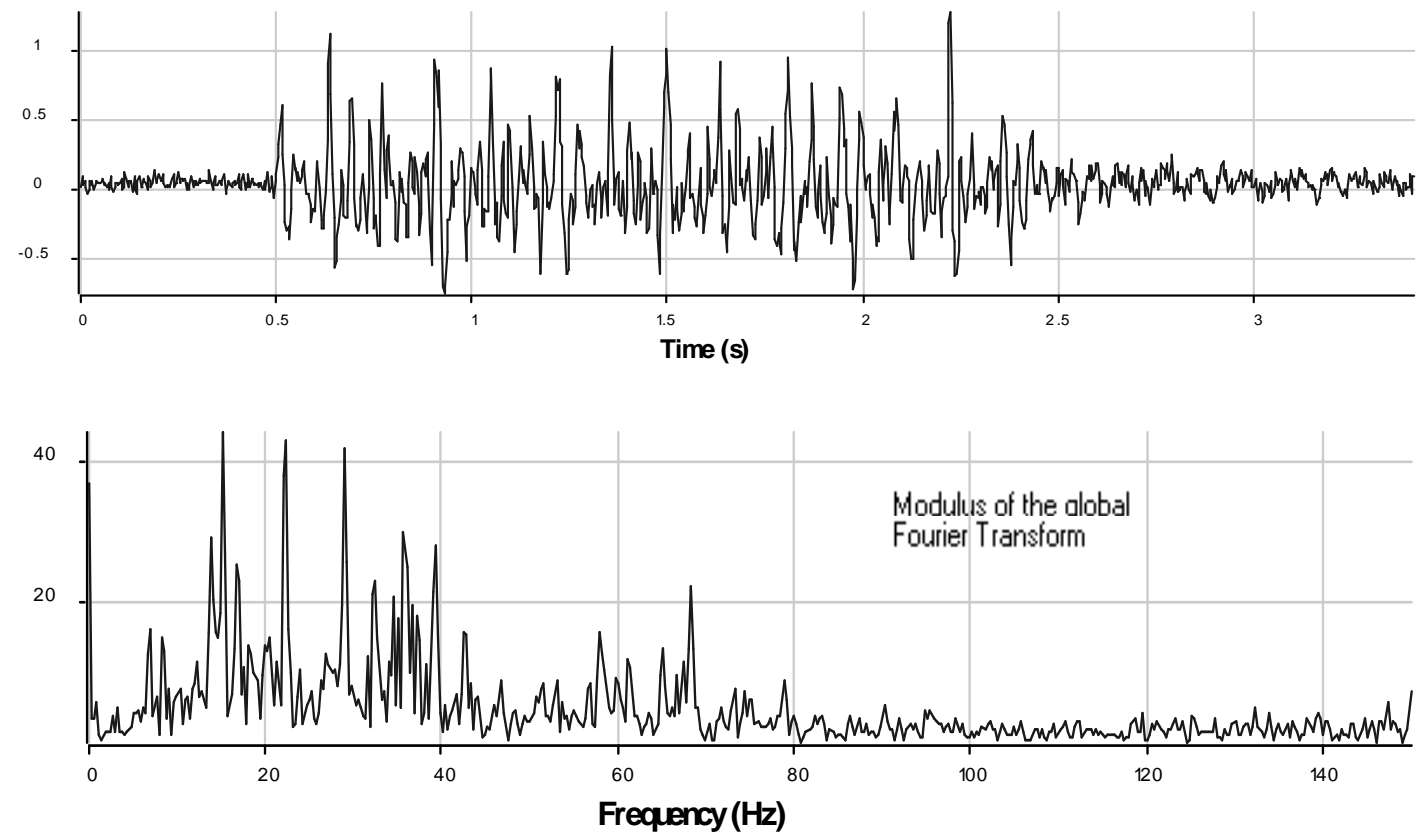

Fig. 3. Acceleration at top of vehicle hanging for a detachable grip. 1024 points were recorded with a sampling frequency of $300 \mathrm{~Hz}$. Lower curve shows the global Fourier Transform.

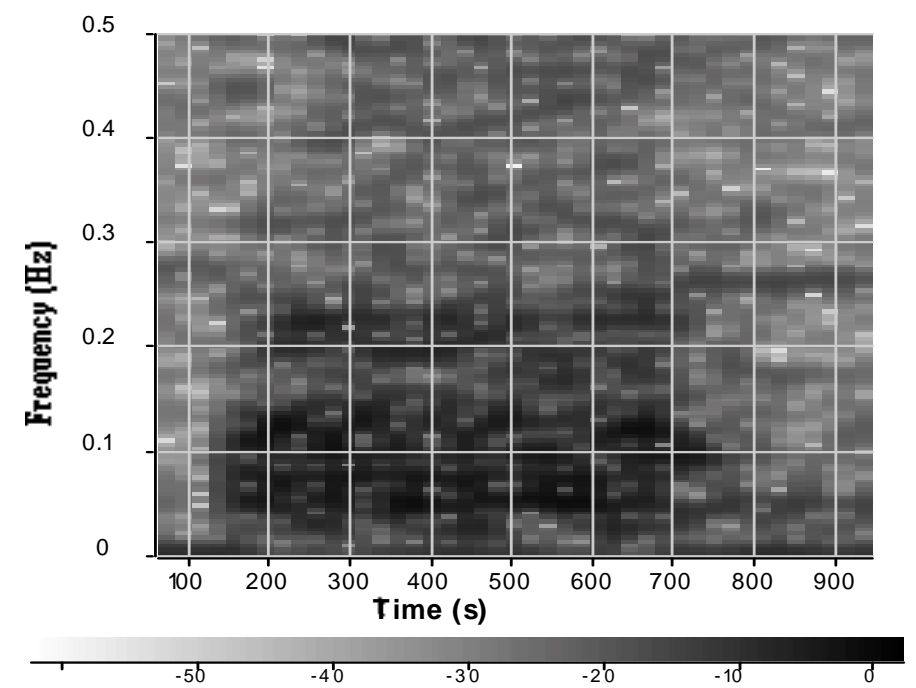

Fig. 4. Spectrogram of acceleration signal of Fig. 3 with Blackman window of width 214 ms (64 points, -3dB band of $7.8 \mathrm{~Hz}$ ).

\section{Stationary correlation Prony method}


Eq. (13) is a sum of delayed damped sinusoids. At each time $t_{m}$, model (14) has common points with the Prony model. Parameter identification of this model has been used since a very long time [PRO 1795]. This Section briefly recalls this stationary approach before dealing with the non stationary one. It will end by the proposition of an original criterion for reducing the sampling effect.

The Prony method models a discrete signal $y[k]$, with sampling period $t_{e}$, from a linear combination of $P$ complex exponential components. Such a model is said to be of order $P$ and the expression for it the noted $\hat{y}[k]$ is

$$
\hat{y}[k]=\sum_{p=1}^{P} a_{p} \cdot z_{p}^{k-1} \quad \forall k=1, N
$$

where $a_{p}$ is the complex amplitude for mode $p, a_{p}=A_{p} . e^{j \Phi_{p}}, z_{p}$ is the complex pole for mode $p$, $z_{p}=e^{-\alpha_{p} t_{e}} \cdot e^{2 \pi j f_{p} t_{e}}$, and $N$ is the number of discrete values in the signal.

\subsection{The two steps of Prony method}

The $\mathrm{z}$ transform of the model, denoted $\hat{Y}(z)$, can be written as

$$
\hat{Y}(z)=\sum_{p=1}^{P} \frac{a_{p}}{1-z_{p} \cdot z^{-1}}=\frac{\sum_{p=0}^{P-1} \beta_{p} z^{-p}}{\sum_{p=0}^{P} \eta_{p} z^{-p}}
$$

where $\beta_{p}, \eta_{p}$ are real coefficients and $\eta_{0}=1$. From this, it will be observed that the model may be considered as the transfer function of an ARMA filter which have $P$ AR complex poles $z_{p}$ and $(P-1)$ MA zeros [SHA 01]. MA and AR part can be written as polynomials, which inverse Fourier Transform leads to

$$
\sum_{p=0}^{P} \eta_{p} \hat{y}[k-p]=\sum_{p=0}^{P-1} \beta_{p} \delta[k-p] \forall k=1, N
$$

When time index $k>P,(k-p)$ is strictly positive and the right-handed term of Eq. (20) is zero. Thus, for $k>P$, we get an equation where differences are function of AR coefficients $\eta_{p}$

$$
\sum_{p=0}^{P} \eta_{p} \hat{y}[k-p]=0 \quad \forall k=P, N
$$

Eq. (18) involves the determination of $2 P$ complex parameters, namely the $P$ poles $z_{p}$ and the $P$ amplitudes $\eta_{p}$. The simultaneous estimate of these parameters using a least-squares method is a non-linear process [BRE 86]. Eq. (21) 
allows splitting the resolution process into two steps. In the first step we get an estimate of the AR coefficients $\eta_{p}$ by a least-squares method. The denominator of Eq. (19) forms a polynomial whose roots are the complex poles $z_{p}$. The second step leads to amplitudes $a_{p}$ by using a least-squares method, this step being linear. So the originality of Prony's method is to put the non-linearity into the determination of the roots of a complex polynomial. A brief reminder of the exact solution, when the number of points in the signal equals $2 \mathrm{P}$, and the least-squares one, where the number of points is greater, are respectively given in Appendices A and B.

\subsection{Others identifications for a noisy model}

The method is particularly sensitive to additive white noise, as Prony model does not introduce any noise. So, the performances degrade when the signal to noise ratio (SNR) decreases. If we have an important added noise, the AR approximation (B.3) still degrades the performances [DON 68], [BLA 75]. Many developments allow overcoming this difficulty. Let us quote the calculation of coefficients from signal space only (i.e. separated from noise space) [KUM 82], [KUM 83], [HEN 81]. Higher order statistics can be used when the noise is gaussian [PAP 90]. Specific models were proposed for coloured noise [SAT 78], [NEH 82], [SAK 86]. In [YOU 01], a threshold on wavelets coefficient eliminate a part of the noise. On a general point of view of rational system response identification without $a$ priori links between poles and zeros, Steiglitz et Mc Bride proposed an identification algorithm based on an iterative calculation using Kalman filters [STE 65], [STO 81].

All these attempts assume that system input is a white noise. Shaw proposed an approach based on an ARMA determinist model, with a pulse input. This model is very suitable in mechanics. The error criterion is then non linear and the solution is reached using iterations [SHA 94], [SHA 01].

For modeling electromechanical oscillations, Sanchez and Chow empirically compare Prony method with a singular value decomposition [SAN 99].

\subsection{A particular case: Prony Correlation}

In Appendix B, it is shown that the first stage of Prony's method is a signal approximation by an ARMA model. Many model parameters estimation have been suggested in the literature [CAS 02], [MAR 87], [BLA 75], [DON 68], [KUM 86]. However, we will not present them here. Nevertheless, we want to point out a specificity of the AR with respect to the autocorrelation function. In the case of an AR or ARMA model, the autocorrelation function keeps the poles unchanged. Indeed, an estimation $\hat{\gamma}_{y y}[k]$ of the autocorrelation of signal $y[k]$ can be defined by 


$$
\hat{\gamma}_{y y}[k]=\frac{1}{N} \sum_{n=1}^{N-k} y[n] \quad y[n+k] \quad \forall k=0, N-1
$$

Let us insert Eq. (B.3) for AR model or Eq. (B.1) for ARMA model at index $(n+k)$ into Eq. (22). Remembering that $e_{l s}[k]$ from Eq. (B.3) is a white noise, we get in a matrix notation

$$
\boldsymbol{\Gamma} \boldsymbol{\eta}=\left(\begin{array}{ccc}
\hat{\gamma}_{y y}[P] & \ldots & \hat{\gamma}_{y y}[1] \\
\ldots & \ldots & \ldots \\
\hat{\gamma}_{y y}[N-2] & \ldots & \hat{\gamma}_{y y}[N-1-P]
\end{array}\right)\left(\begin{array}{c}
\eta_{1} \\
\ldots \\
\eta_{P}
\end{array}\right)=-\left(\begin{array}{c}
\hat{\gamma}_{y y}[P+1] \\
\ldots \\
\hat{\gamma}_{y y}[N-1]
\end{array}\right)=-\gamma
$$

The $(N-1-P) \times P$ correlation matrix $\Gamma$ is a Toeplitz matrix, because of the same values along each diagonal. These equations lead to an important conclusion: the autocorrelation function of an AR signal is also an AR model, with the same coefficients. Furthermore, when the delay is greater than the model order $P$, the autocorrelation is independent of the noise if it is a broadband noise. So, it appears to be interesting to apply the Prony method on the autocorrelation function of the signal rather than on the signal itself (see Appendix C). The resolution of the first Prony step needs inversion of the correlation matrix $\mathbf{C}$. The second step needs inversion of the Vandermonde matrix $\mathbf{Z}$ defined in Eq.(C.3).

\subsection{Sampling influence on $\Gamma$ and $\mathrm{Z}$ conditioning}

Prony resolution involves two linear systems (see Eqs. (23) and Erreur ! Source du renvoi introuvable.). For the solution to be less sensitive to perturbations, it is important to optimise the matrix conditioning. We want to present here a simple and original operating mode. Solving a linear system requires one to invert a matrix, either explicitly or implicitly. The inverse of a matrix can be written in terms of the inverse of its singular values. If the smallest singular value is close to 0 , the solution will be sensitive to any small perturbation [GOL 83]. The sensitivity of a matrix $\boldsymbol{\Gamma}$ can be measured by its conditioning $\kappa_{\Gamma}$ defined by

$$
\kappa_{\Gamma}=\|\Gamma\|\left\|\Gamma^{-1}\right\|
$$

where $\|\cdot\|$ is the matrix norm [GOL83]. A matrix is correctly conditioned when $\kappa_{\Gamma}$ is weak. Whichever the norm may be, $\kappa_{\Gamma}>1$. So, it may be interesting to look at the inverse $\kappa_{\Gamma}^{-1}$, which is limited to the range $[0,1]$. Thus, $\kappa_{\Gamma}^{-1}$ is close to 1 for a good conditioning. When we choose the norm $2, \kappa_{\Gamma}$ can be written as the ratio of the largest singular value $\lambda_{\max }(\Gamma)$ to the smallest one $\lambda_{\min }(\Gamma)$. So, 


$$
\kappa_{\Gamma}^{-1}=\frac{\lambda_{\min }(\Gamma)}{\lambda_{\max }(\Gamma)}
$$

For $\Gamma$ defined in Eq. (23), $\kappa_{\Gamma}^{-1}$ decreases when the sampling rate $f_{e}$ increases. This can be explained as follows. In a Toeplitz matrix, when $f_{e}$ increases, the column vectors become more and more dependent, its rank decreases and the matrix becomes less and less invertible [HUN 72]. On the other hand, for small values of $f_{e}, \kappa_{\Gamma}^{-1}$ goes up with $f_{e}$. Therefore, there is an optimum value of $f_{e}$, which is given by the position of the maximum of $\kappa_{\Gamma}^{-1}$ versus $f_{e}$

$$
f_{e} \quad \text { optimal }=\underset{f_{e}}{\arg \max }\left(\kappa_{\Gamma}^{-1}\right)
$$

Contrary to Fourier based methods, analysing an over-sampled signal with a parametric method decreases the precision of the analysis. Fig. 5 presents results for simulated signals and converges to a well-known result. The analysis of a signal constructed with only one frequency is best when this frequency equals $1 / 4$ of the sampling frequency. The maximum of the curve will be observed when $f_{e} / f_{\max }=4$. When two frequencies are present, the ratio should be close to 3 .

When a real signal has been recorded, it is no longer possible to vary the sampling rate. It can nevertheless be interesting to have a first interpolation of the signal to be closer to the optimum value, mainly if the noise is not negligible. This step will prove to be useful in the shock instant detection proposed in Section 6.2.

As shown in Fig. 5, conditioning of matrix $\mathbf{Z}$ defined in Eq. (C.3) is less sensitive to sampling rate than conditioning of $\boldsymbol{\Gamma}$. Nevertheless, it is important to watch its value as it influences the amplitude calculation, which, in turns, influences, the shock instant detection.
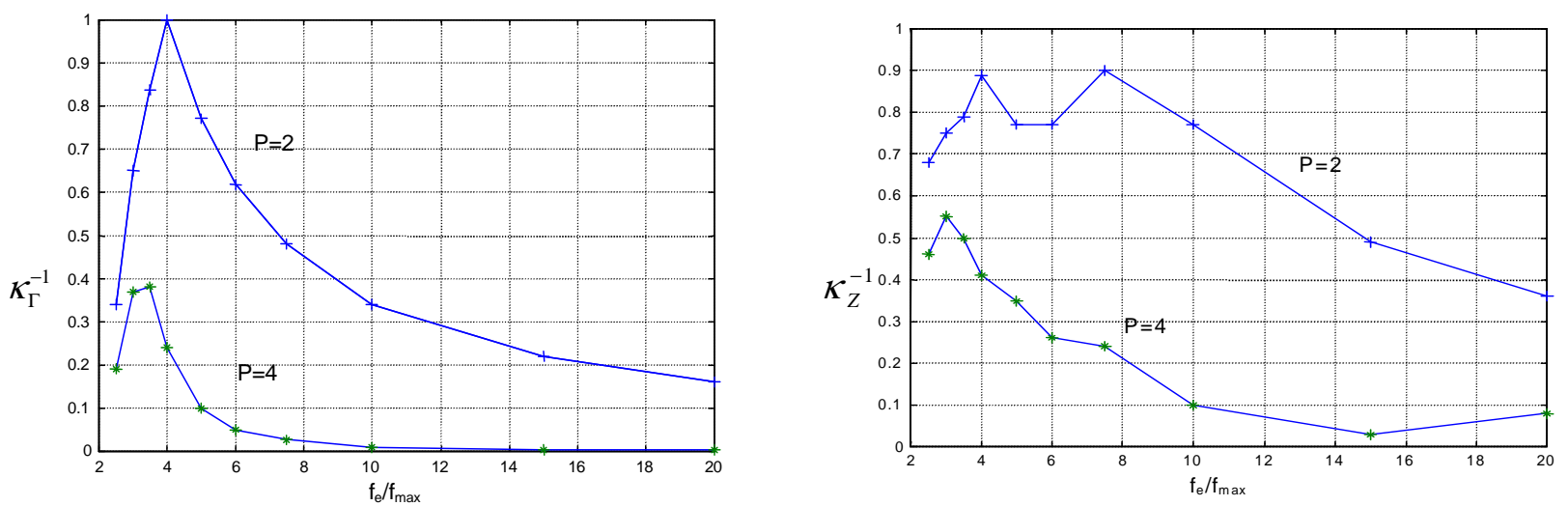

Fig. 5. Sampling Influence on $\boldsymbol{\Gamma}$ conditioning (left) and $\mathbf{Z}$ conditioning (right): $P=2$ corresponds to a simulated signal built with a damped sine. $P=4$, includes two damped frequencies. SNRis $11 \mathrm{~dB}$. 


\section{Non stationary and multishock model}

On many systems, the recorded vibration signals are often the result of very short events, like shocks between different mechanical components, which excite the resonance frequencies of the system. If these events are close to each other, the system responses from each one may interfere with the signal. Assuming a linear and stationary response, the recovery of the excitation signal is called a deconvolution problem [LEE 98], [COM 02]. But in the case of a nonstationary transfer function of the system, or of a variation of the system configuration, another approach is necessary. In this paper, we focus on linear and non-stationary systems whose output signals can be written as a linear combination of exponentially damped sinusoids with different starting instants $t_{m}$. Such signals follow the multishock model as described by Eq. (13). We showed in Section 4 that the limits of the usual time-frequency detection methods are rapidly reached in order to separate close shocks in time. So we propose here a new instant detection based on the PronyCorrelation method applied to a gliding window.

\subsection{Model rupture on analysis window}

Let us first recall [BRI 81] the definition of stationarity. A signal is said to be stationary if there is a simple, analytic and converging relation between its values at different times. In this way, when the window is opened before the beginning of the signal, the signal included in the window cannot be considered as stationary and the Prony method will give poor results.

When the window is opened too early (in phase advance), applying Prony method to this window would lead to important errors. When resolving Eq.(C.1), we would get an error for AR parameters, error which varies as the ratio of the signal power before the shock to the shock response power [ROB 96]. When resolving Eq. (C.4), Yvetot [YVE 96], [YVE 93] showed that amplitude estimation has both a multiplicative bias linked to the model poles and an additive bias linked to the signal samples amplitude before the shock. This bias will be more or less important, depending on what these samples are, either noise or the end of the previous shock response.

On the opposite, if the window is opened after the starting instant of the shock, the estimated parameters will be those of the signal present in the window with a delay which will influence phase and amplitude values in the resolution of Eq.(C.4). With a phase delay $\varphi_{r}$, the amplitude and phase of each mode $p$ becomes equal to $A_{p} e^{-\alpha \varphi_{r} / \Omega} e^{j\left(\varphi_{r}+\phi_{p}\right)}$ instead of $A_{p} e^{j \phi_{p i}}$. Fig. 6 illustrates that sensitivity to window position. 

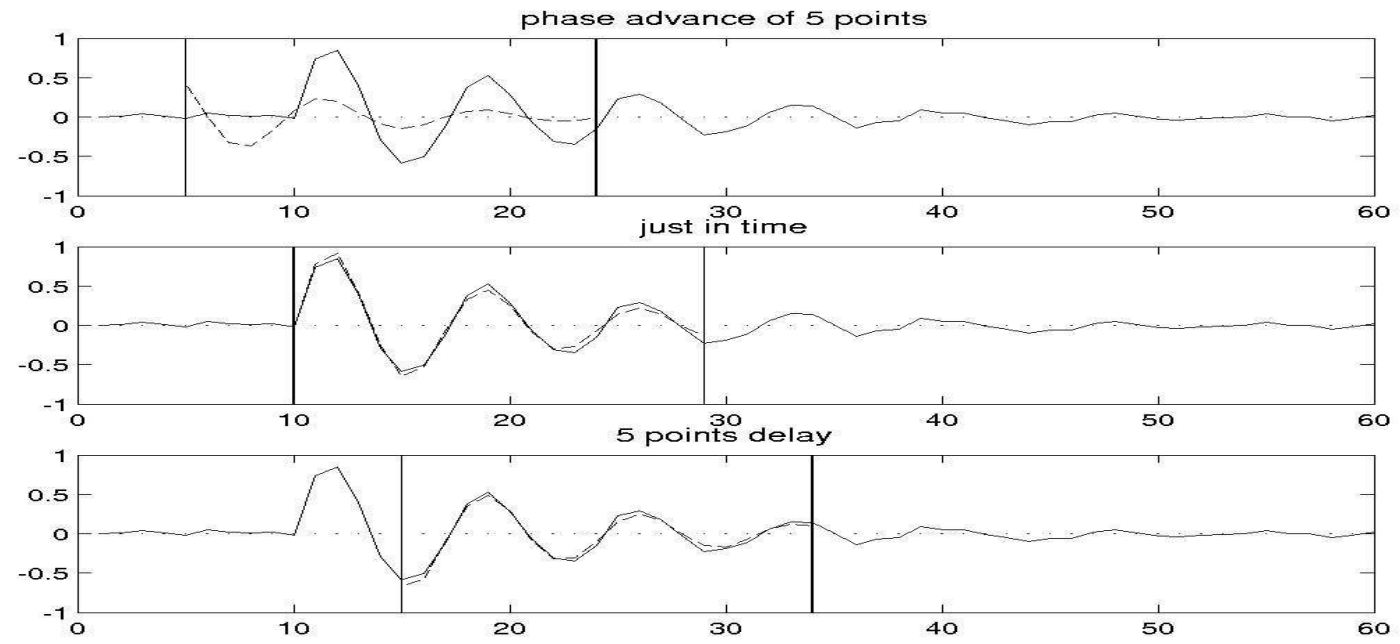

Fig. 6. Sensitivity of PRONY analysis to position of the analysis window: phase advance, just in time and phase delay. The blue curve is the simulated signal ( $\exp (-0.4(t-10)) \cdot \sin (2 \pi 0.14(t-10))$ with te $=1 \mathrm{~s}, S N R=18 \mathrm{~dB}$, the superposed red curve is the estimated Prony model at order $P=2$ with a window size of $N=20$ points (marked by two vertical lines).

\subsection{Shock instants detection: amplitude curve}

To bypass the problem due to observation window position, we first estimate the model rupture instants. Let us note the shock instants as $t_{m}=k_{m} t_{e}$.

Let us first assume that responses to shocks do not overlap. Consider a $N_{f}$ points gliding window, $N_{f}$ being of the order of the point number that separates the shocks. Let $k_{f}$ be the first time index of this window.

When $k_{m} \leq k_{f} \leq k_{m+1}-N_{f}$, the signal on which the window is open has a phase delay $\varphi_{r}=k_{f} \Omega_{p}$ (see Section 6.1).

With an origin fixed at the first point of the window and with $0 \leq k \leq N_{f}-1$, the model $y_{f}[k]$ can be written as

$$
y_{f}[k]=\sum_{p=1}^{P} A_{m p} e^{-\alpha_{p}\left(k+k_{f}-k_{m}\right) t_{e}} e^{\Omega_{p}\left(k+k_{f}-k_{m}\right) t_{e}+\phi_{m p}}
$$

Let $A_{p}\left[k_{f}\right]$ be the amplitude for mode $p$ in the analysis window. We can write it as

$$
A_{p} \quad\left[k_{f}\right]=A_{m p} e^{-\alpha_{p}\left(k_{f}-k_{m}\right) t_{e}}
$$

Eq. (28) shows that, in the window, each mode amplitude exponentially depends on $k_{f}$, the window position, and is maximum at $k_{f}=k_{m}$. 
When $k_{m+1}-N_{f}+1 \leq k_{f} \leq k_{m}-1$, the window is open before the signal occurs (phase advance) and it contains a model rupture. Amplitude estimations will be perturbed during a time corresponding to the window width $N_{f}$. Given that the model parameters are estimated in two separate steps, poles estimation from Eq. (A.2) then amplitudes from Eq. (C.4), it is possible to choose different window size for each step. This processing is interesting since our interest in the first step is the detection, so the sensibility to a variation rather than an accurate estimation. Let $N_{f}$ bis be the window length in the second step. If we choose $N_{f}$ bis $<<N_{f}$, amplitude estimation will be perturbed on a lesser extent. Fig. 7 presents two Prony analyses, one with identical window size for both steps and the other with different sizes. Choosing $N_{f}$ bis $=2 P$ seems to be a good compromise.

Therefore, when the window glides point by point over a multi-pulse signal, the amplitude $A_{p}\left[k_{f}\right]$ is a succession of decreasing exponentials and the maxima of the curve indicate a synchronism between the shock and the opening of the window. This is true for each mode $p$. As there is no reason to focus more onto one mode than another, we will focus onto the sum of amplitudes for all modes. Thus, the shocks instants $k_{m}$ will be detected with the arguments of amplitude sum maxima

$$
\hat{k}_{m}=\underset{k_{f}}{\arg \max } \sum_{p=1}^{P} A_{p}\left[k_{f}\right] \text { with } A\left[\hat{k}_{f}\right]>\varepsilon_{1} \text { and } \varepsilon_{1}=\lambda \sigma
$$

with $\sigma$ the noise standard deviation, which can be estimated from the spectrogram using a maximum likelihood method [HOR 02] for instance, and $\lambda$ an a priori fixed real number.
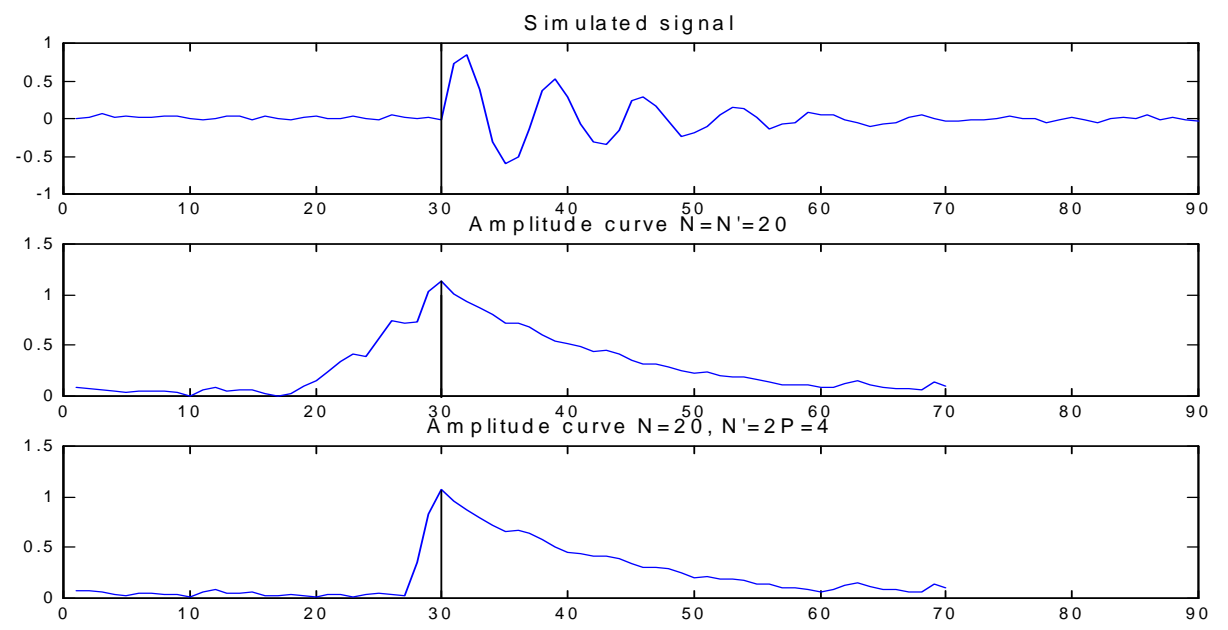

Fig. 7. Amplitude curve estimated from the simulated signal in top figure. Middle: amplitude curve estimated with windows of the same size in the two Prony steps $\left(N_{f}=N_{f b i s}=20\right.$ pts). Lower: amplitude curve with different sizes $\left(N_{f}=20, N_{f b i s}=2 P=4\right.$ pts $)$. 
In order to decrease false alarm probability, we add a second constraint by detecting exponential decreases after each maxima $k_{m}$. Between two maxima $k_{m}$ and $k_{m+1}$, we approximate an exponential function with amplitude $\hat{A}_{k_{f}}$ and damping factors $\hat{\alpha}_{k_{f}}$ by least-squares and retain only maxima $k_{m}$ which residue's power is under a fixed threshold $\varepsilon_{2}$. So, we have

$$
\frac{\sum_{k_{f}=k_{m}}^{k_{m+1}}\left|\sum_{i=1}^{P} A_{p}\left[k_{f}\right]-\hat{A}_{k_{f}} \cdot e^{-\hat{\alpha}_{k_{f}} \cdot\left(k_{f}-k_{m}\right) t_{e}}\right|^{2}}{\sum_{k_{f}=k_{m}}^{k_{m+1}}\left|\sum_{p=1}^{P} A_{p}\left[k_{f}\right]\right|^{2}}<\varepsilon_{2}
$$

We should also note that, even if responses to shocks overlap, a correct detection is still possible if we assume that the same frequencies are excited by the shocks, with the same damping factor. If this is the case, it can easily be shown that each portion of the amplitude curve is a decreasing exponential, whatever the distance between two shocks. For instance, for two shocks at times $k_{1}$ and $k_{2}$, with the same pulsation $\Omega$, the same damping factor $\alpha$ and $t_{e}=1 s$ for sake of simplicity, we have

$$
y[k]=A_{1} e^{-\alpha\left(k-k_{1}\right)} e^{j \Omega\left(k-k_{1}\right)+j \phi_{1}} U\left[k-k_{1}\right]+A_{2} e^{-\alpha\left(k-k_{2}\right)} e^{j \Omega\left(k-k_{2}\right)+j \phi_{2}} U\left[k-k_{2}\right]
$$

The area under concern here is $k>k_{2}$ where we have interferences between both vibrations

$$
y(k)=e^{-\alpha\left(k-k_{2}\right)}\left(A_{1} e^{-\alpha\left(k_{2}-k_{1}\right)} e^{j \Omega\left(k-k_{1}\right)+j \phi_{1}}+A_{2} e^{j \Omega\left(k_{1}-k_{2}\right)+j \phi_{2}}\right) \text { for } k>k_{2}
$$

Eq. (32) can be written as

$$
y[k]=A_{k_{2}} e^{-\alpha\left(k-k_{2}\right)} e^{j\left(k-\Phi_{k_{2}}\right)}
$$

where $A_{k_{2}}$ and $\Phi_{k_{2}}$ depend on $k_{1}, k_{2}, \phi_{1}$ and $\phi_{2}$. According to these parameters, amplitude $A_{k_{2}}$ varies between two limits

- $\quad A_{k_{2} \max }=A_{1} e^{-\alpha\left(k_{2}-k_{1}\right)}+A_{2} \quad$ when interferences are constructive;

- $\quad A_{k_{2} \min }=\left|A_{1} e^{-\alpha\left(k_{2}-k_{1}\right)}-A_{2}\right| \quad$ when interferences are destructive.

Fig. 8 and 9 illustrate this situation for a signal made of three shocks: the first two interfere constructively, whereas the last two interfere destructively. In Fig. 8, without noise, the last shock could be detected. But, in Fig. 9, 5 false alarms occur in parts of the signal where the SNRis weak ( $6,5 d B$ for the $3^{\text {rd }}$ shock). In the next Section, we show the limits of the detector for destructive interference when noise increases. 

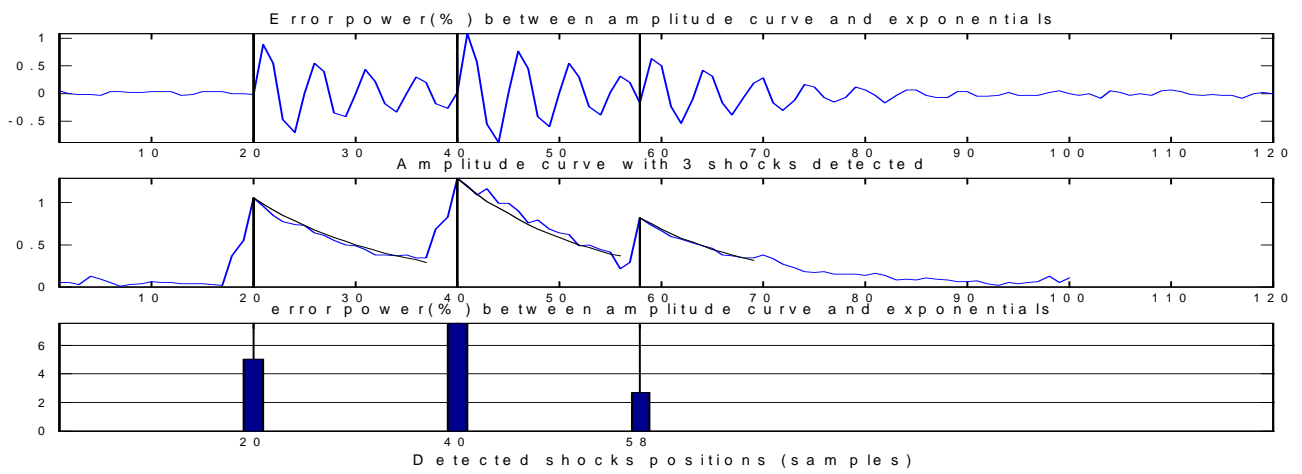

Fig. 8. Amplitude curve and detected shocks (middle) on a simulated signal (upper) where the first two shocks interfere constructively, whereas the last two interfere destructively. Global error for each shock (lower).

$N_{f}=20$ points, $N_{f b i s}=2 P=4$ points, $\varepsilon_{1}=A \max / 5, \varepsilon_{2}=0.1$.
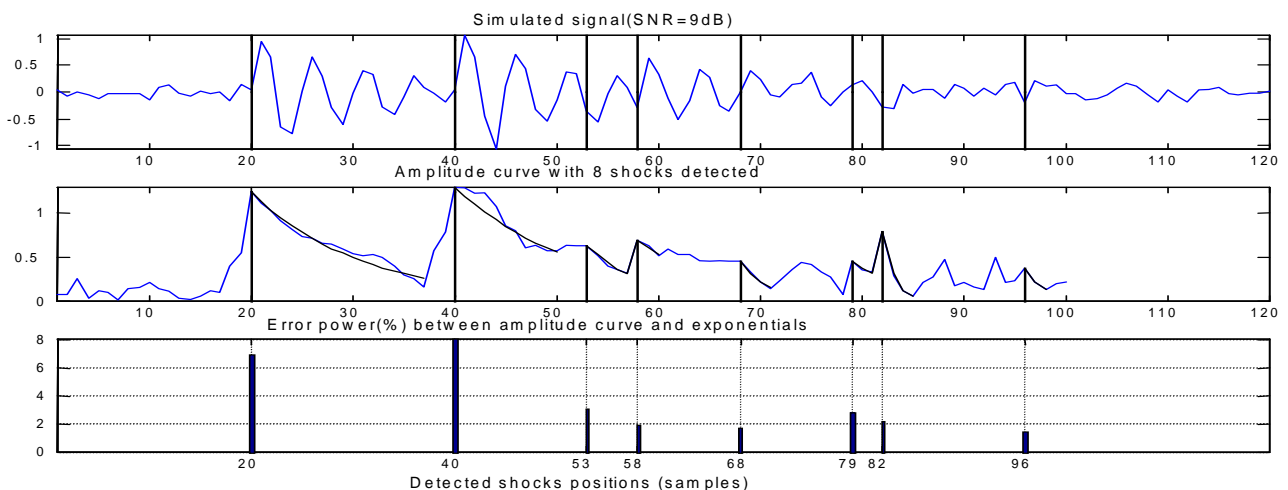

Fig. 9. Amplitude curve and detected shocks (middle) on a simulated signal (upper) where local SNR is $12 \mathrm{~dB}$ for the $1^{\text {st }}$ shock, $14 \mathrm{~dB}$ for the $2^{\text {nd }}$ and $6,5 \mathrm{~dB}$ for the $3^{\text {rd }}$ one. Global error for each shock (lower). $\varepsilon_{1}=A_{\max } / 5, \varepsilon_{2}=0.1$.

\subsection{Instant detector performance}

Fig. 10 presents the Receiver Operating Characteristics or ROC. These curves show the detection probability $P d$ versus false alarm probability $P f$, for various SNRvalues whereas conditional probabilities $P d$ and $P f$ are defined as

$$
P_{d}=P\left(\hat{k}_{m}=k_{1} \mid k_{1}\right) \quad \text { and } \quad P_{f}=P\left(\hat{k}_{m} \neq k_{1} \mid k_{1}\right)
$$

The threshold $\varepsilon_{2}$ is the varying parameter. Given that threshold $\varepsilon_{1}$ is less critical, only two values of $\varepsilon_{1}$ are chosen.
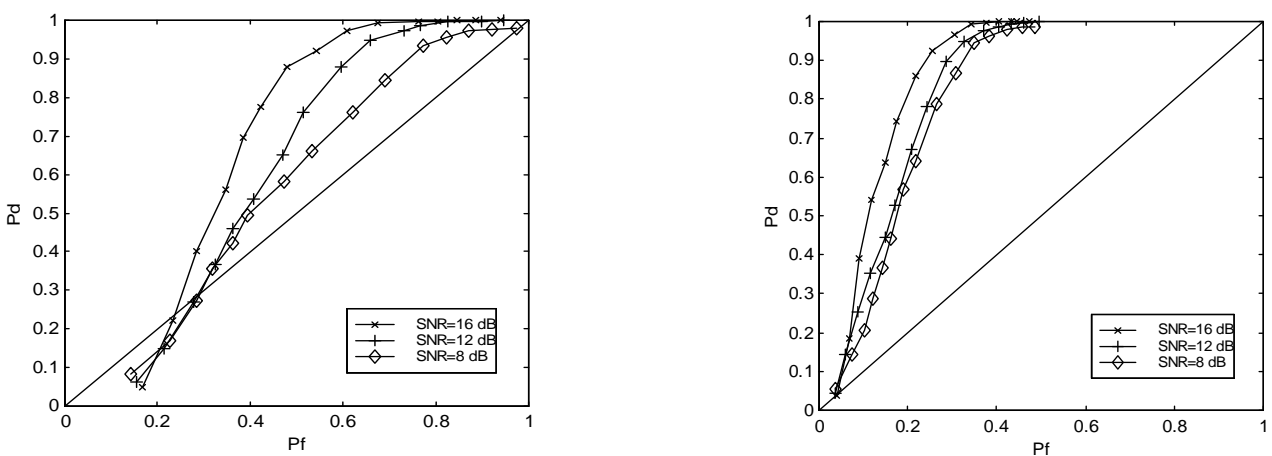

Fig. 10. ROC (Pd versus Pf) for instant detection of a one shock simulated signal with $\varepsilon_{1}=3 \hat{\sigma}($ left $)$ and $\varepsilon_{1}=6 \hat{\sigma}$ (right). 
Fig. 11 presents a simulated three shocks signal with same amplitude and response for each shock but a varying delay between shocks. In case of a constructive interference, as 2 and 3 periods (upper and bottom curves of Fig. 11), the ROC shows a similar detection to that for one single shock of Fig. 10. The middle curve of Fig. 11 represents the ROC for a destructive interference ( 2.5 periods). In that case, performance is unacceptable.
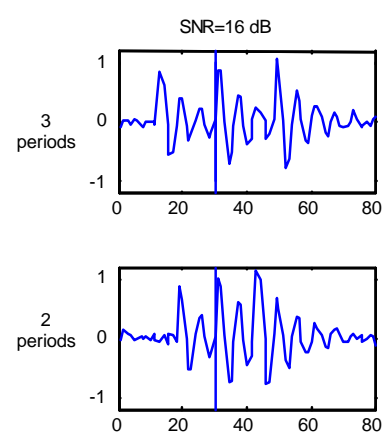

Constructive interferences
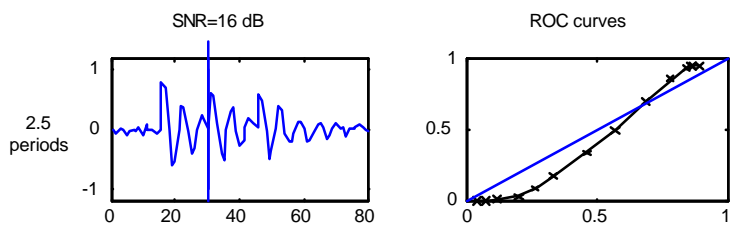

Destructive interferences

$-1$
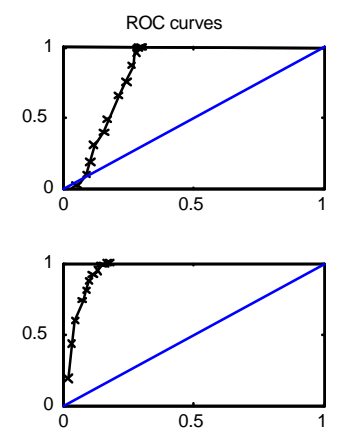

Fig. 11. Detection performance for a simulated 3 shocks signal with various delays between shocks: 3 and 2 periods of the signal for constructive interferences, 2.5 periods of the signal for destructive interferences. SNR $=16 \mathrm{~dB}$. For each period, left curve shows the signal and right curve the ROC with $\varepsilon_{1}=6 \hat{\sigma}$.

\subsection{Time-frequency Prony}

In previous Section, we assumed that either shock responses do not interfere or the local response is stationary. This allows us to choose a wide $N_{f}$ for good poles estimation and a short size $N_{f b i s}<N_{f}$ to improve shock detection and separation. Thus, both estimation and detection constraints are filled. In a second stage, shock instant detection allows a temporal segmentation of the signal. In a third stage, we apply gliding Prony's method to each segment and we finally reconstruct a whole signal model. The overall method structure is shown in table 1 .

\begin{tabular}{|l|}
\hline $\begin{array}{l}\text { Prony's model applied to a gliding-window: } \\
\text {-first step: size } N_{f} \text { fixed } \text { a priori (about } 3 \text { periods) } \\
\text {-second step: size } N_{f b i s}<N_{f} \text {, order } P \text { a priori }\end{array}$ \\
\hline $\begin{array}{l}\text { Shock instant detection on the amplitude curve: } \\
\text { detection of exponential decreasing after maxima } \\
\text { using detection criterion (Eq. } 4 \text { ) }\end{array}$ \\
\hline $\begin{array}{l}\text { Whole signal modeling: Prony's model applied to each } \\
\text { segment between shocks instants or on a minimum size } \\
\text { of } N_{f} \text { points. }\end{array}$ \\
\hline
\end{tabular}

Table 1. Time-Frequency Prony Algorithm

Fig. 12 shows comparison between the analyzed signal and the reconstructed signal for signal of Fig. 8. Model error is calculated from the ratio of the residue power to the signal power. 

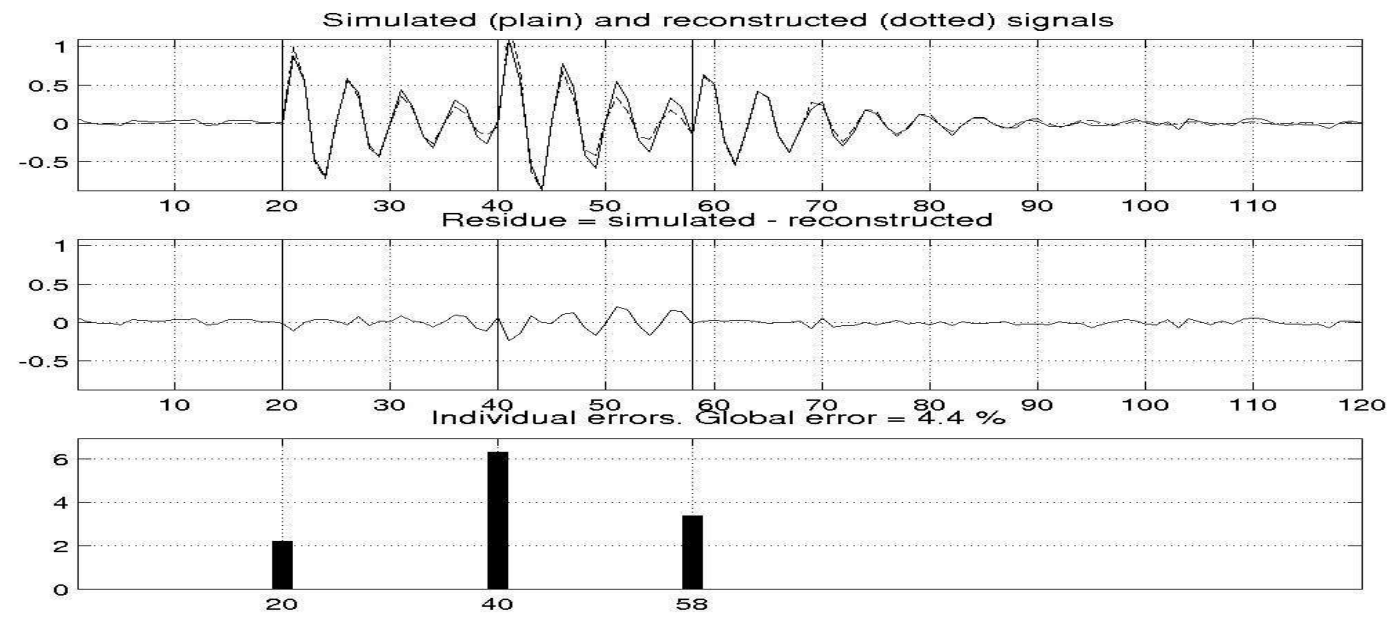

Fig. 12. Simulated signal superposed on the reconstructed one by time-frequency Prony method (upper). Error versus time (middle). Global error for each shock (lower).

\section{Application to a chairlift acceleration signal}

In order to illustrate the method that we presented here, we are going to present results on the rope chairlift system mentioned in Section 3. First, in Section 7.1, we design an acceleration model derived from Eq. (16), parameters being estimated from mechanical knowledge. We call this model a mechanical model to set it apart from the Prony model estimated by the method we propose. Then, in Section 7.2, we analyze a recorded signal.

\subsection{Analysis of a mechanical model}

In the model design, we will assume that only the degree of freedom of the sub-rocker in contact with the grip will be excited (see Section 3). The vibration frequency of this sub system can easily be calculated and is found to be around 30 Hz. This frequency is rather stable for standard installations, because the legal rules are very strong and only a little liberty is given to the tension of the rope. From many measurements, we observed that the damping factor turns out to be around $50 s^{-1}$. From what precedes, we can work out the induced acceleration to the chair. The real rocker impulse response can be calculated from Eq. (8) with $P=2$. It is then possible to calculate the overall response when the grip runs under the rocker. Under the form of acceleration, we have to use Eq. (16). The rocker is excited by excitation (10). Figs. 13, 14 and 15 show the accelerometric response we obtain and the results of the time-frequency Prony method at 1,2 and $4.16 \mathrm{~m} / \mathrm{s}$, respectively.

In these figures, the upper curve is the mechanical model, the second one shows the reconstructed signal on which vertical lines represent the detected shocks. The frequency, damping factor, amplitude and phase value evolution are then presented versus time. On the last curve, the vertical lines shows the simulated excitation model. 
At speed 1m/s (Fig. 13), the window width for the instant detection is fixed to the minimum gap between two shocks ( $N_{f}=10$ ). Every shock instant is well detected. The estimated frequencies and damping factors remain quite stable and correspond to the simulated ones. Amplitudes and phases also correspond to the model: we recover the shocks with amplitude 0.75 and phase $+\frac{\pi}{2}$ and those with amplitude 0.5 and phase $-\frac{\pi}{2}$.

At speed 2m/s (Fig. 14), $N_{f}$ is also fixed to 10 and cannot be fixed to a lower value, since it is about the period of the vibration frequency. Thus, the selected window width for the instant detection is superior to the minimum gap between two shocks. Where the shocks are too close to each other, we observe a bad detection. As a consequence, parameter estimation of these close shocks is less accurate. The other ones are correctly detected and estimated.

At speed $4.16 \mathrm{~m} / \mathrm{s}$ (Fig. 15), which is the speed of the real experimentation presented in the next Section, $N_{f}$ is also fixed to 10 and the reconstruction error increases again due to the same problem. It is important to notice that this situation is very critical (see Fig. 1d also).

24 shocks, reconstruction error $(\%): 1.135$

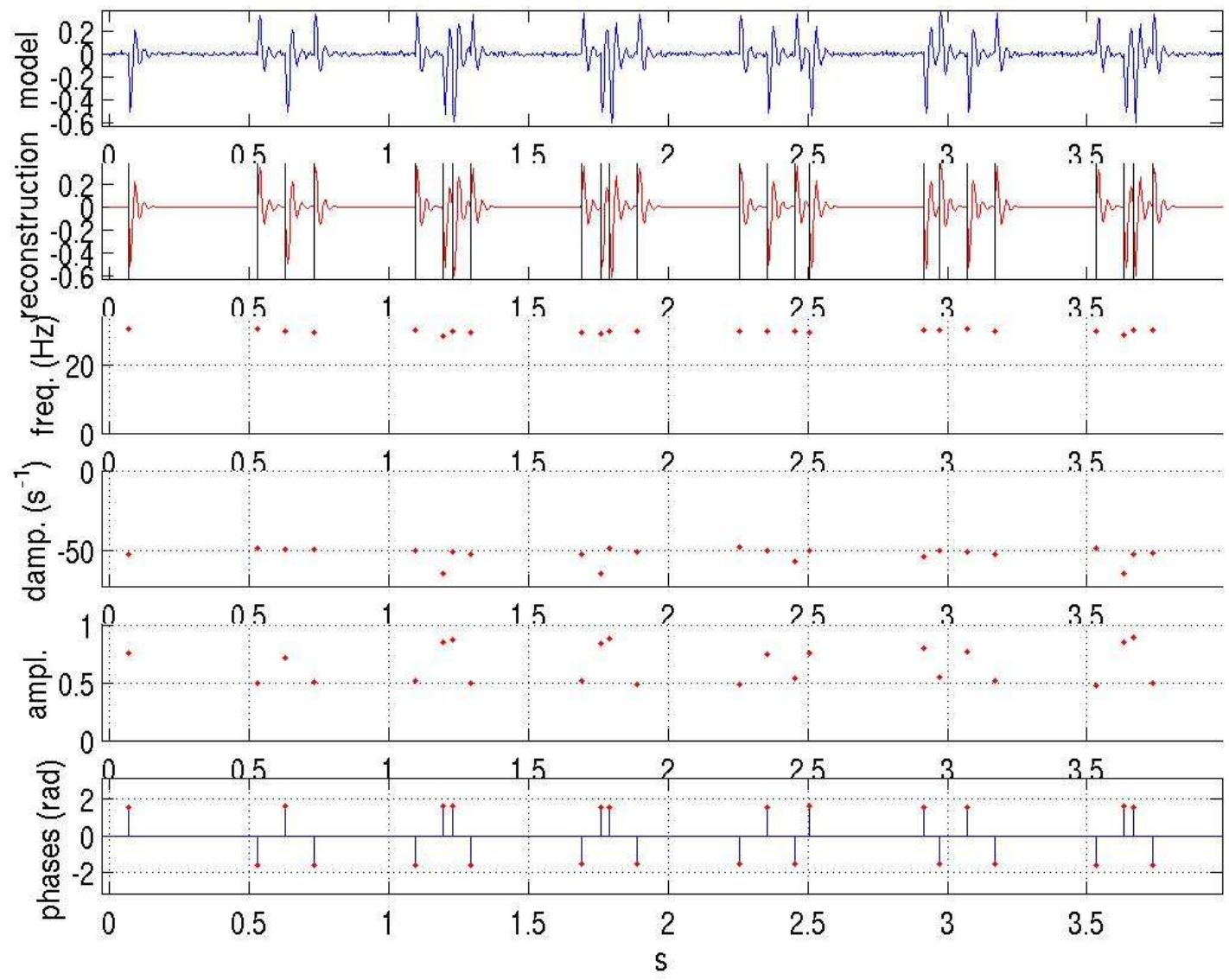

Fig. 13. Time-frequency Prony method applied to a mechanical model of the interaction grip / 12 wheel rocker at speed $1 \mathrm{~m} / \mathrm{s}$. From up to bottom: mechanical model with frequency $30 \mathrm{~Hz}$, damping factor $50 \mathrm{~s}^{-1}$ and $S N R=20 \mathrm{~dB}$; detected shock instants (vertical lines) and signal reconstruction by time-frequency Prony $\left(P=2, N_{f}=10\right)$; estimated frequencies versus time; estimated damping factors 
versus time; estimated amplitudes versus time; estimated phases versus time. On the last curve, the vertical lines indicate the shock instants of the excitation model.

24 shocks, reconstruction error (\%): 7.4638

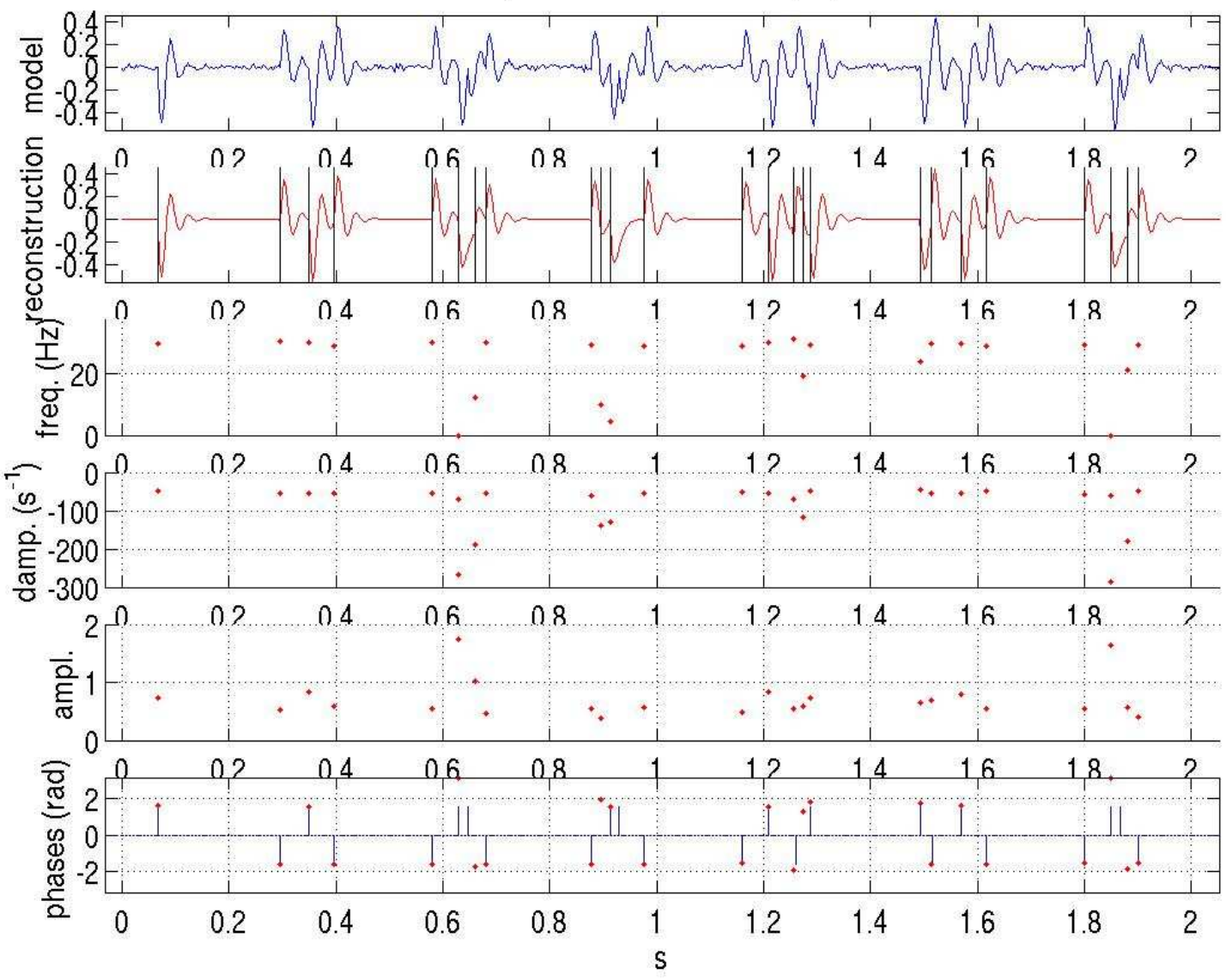

Fig. 14. Time-frequency Prony method applied to a mechanical model of the interaction grip / 12 wheel rocker at speed $2 \mathrm{~m} / \mathrm{s}$. From up to bottom: mechanical model with frequency $30 \mathrm{~Hz}$, damping factor $50 \mathrm{~s}^{-1}$ and $S N R=20 \mathrm{~dB}$; detected shock instants (vertical lines) and signal reconstruction by time-frequency Prony $\left(P=2, N_{f}=10\right)$; estimated frequencies versus time; estimated damping factors versus time; estimated amplitudes versus time; estimated phases versus time. On the last curve, the vertical lines indicate the shock instants of the excitation model.

\subsection{Analysis of a chairlift model}

Let us now analyse a real signal, namely the acceleration measurement at the top of a hanging chair in a detachable 4seats chairlift. Signal is recorded when cable is at speed $4.16 \mathrm{~m} / \mathrm{s}$. The rope wearing induces a vibration, which is better to filter before analysing the signal. This vibration frequency can be calculated from the rope wearing step and the velocity. Before processing, the real signal is so filtered by a low-pass filter with a cut-off frequency equal to $50 \mathrm{~Hz}$.

Fig. 16 shows time-frequency Prony results on the filtered real signal. The model order here also is $\mathrm{P}=2$ ( 1 frequency in real signal analysis). The mean value of the estimated frequencies is $26 \mathrm{~Hz}$ and their standard deviation $7 \mathrm{~Hz}$. The fluctuation of the estimated parameters can be explained by the critical case of this situation: high speed and shocks very close to each others, as in the simulation (see Fig. 15). 
48 shocks, reconstruction error (\%) : 8.7

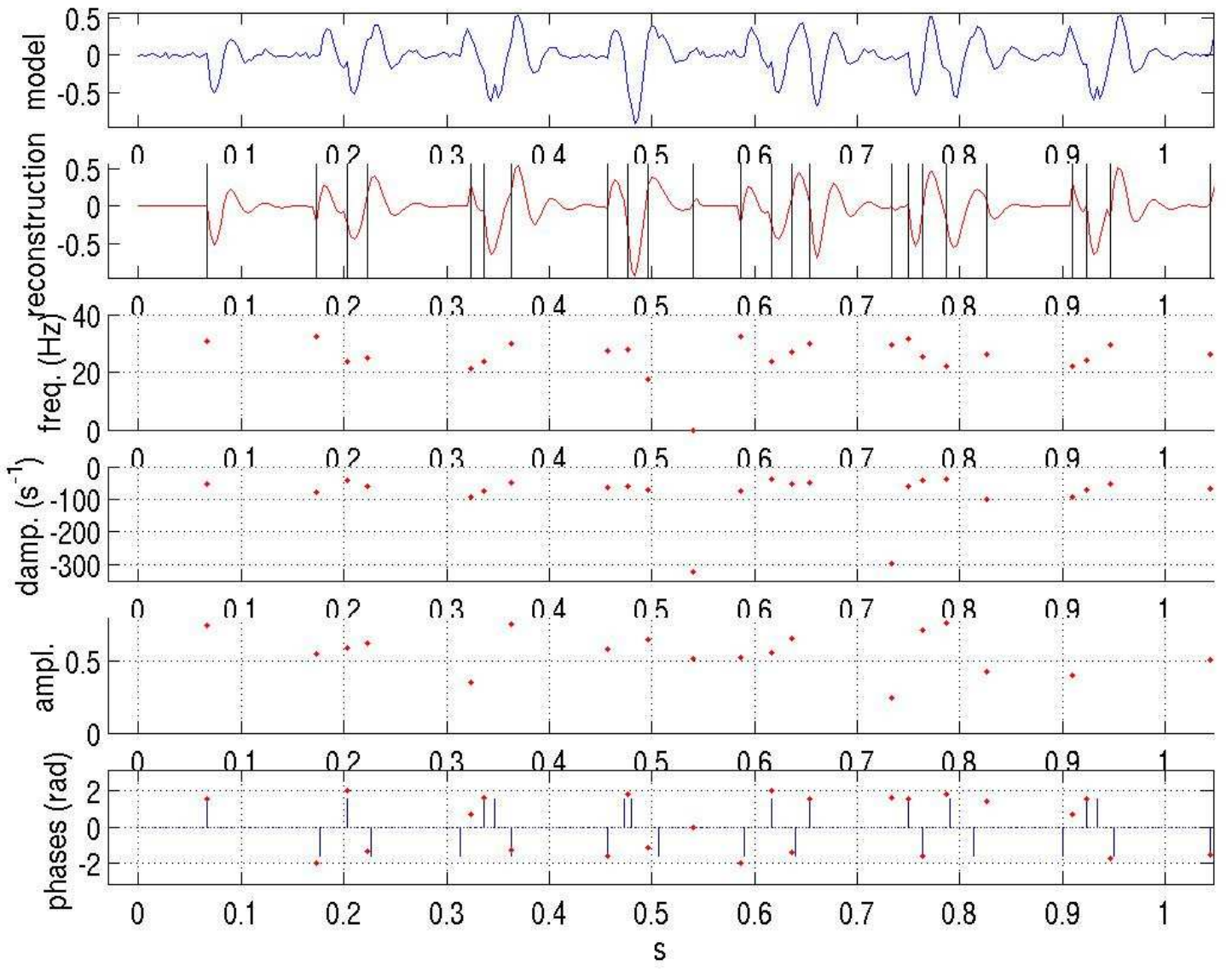

Fig. 15. Time-frequency Prony method applied to a mechanical model of the interaction grip / 12 wheel rocker at speed $4.16 \mathrm{~m} / \mathrm{s}$. From up to bottom: mechanical model with frequency $30 \mathrm{~Hz}$, damping factor $50 \mathrm{~s}^{-1}$ and $\mathrm{SNR}=2 \mathrm{~d} \mathrm{~dB}$; detected shock instants (vertical

lines) and signal reconstruction by time-frequency Prony $\left(P=2, N_{f}=10\right)$; estimated frequencies versus time; estimated damping factors versus time; estimated amplitudes versus time; estimated phases versus time. On the last curve, the vertical lines indicate the shock instants of the excitation model.

\section{Conclusion}

Understanding the chairlift behaviour opens the way to a predictive model, a situation which is indeed a safer situation, as it allows to calculate dynamic strains in the vehicles (and also in the towers). This allows checking the equipment reliability before construction, and also to make sure that the equipment will pass all legal tests it has to undergo.

On a signal processing point of view the need for a model of a mechanical system allowed us to propose a new method for shock detection and transient signal analysis. We succeeded in detecting very close shocks, even though the responses were overlapping. The detection method is based on estimation of the physical parameters of a system. The good performance can be due to the fact that the model is close to the physical reality when vibrations are involved. Being a non-stationary analysis, the model allows a large variety of applications. So, it could be applied to many 
vibration situations. An interesting progress would be to include in our process a deflation step. We are currently working on this problem.

48 shocks, reconstruction error $(\%): 19.3$

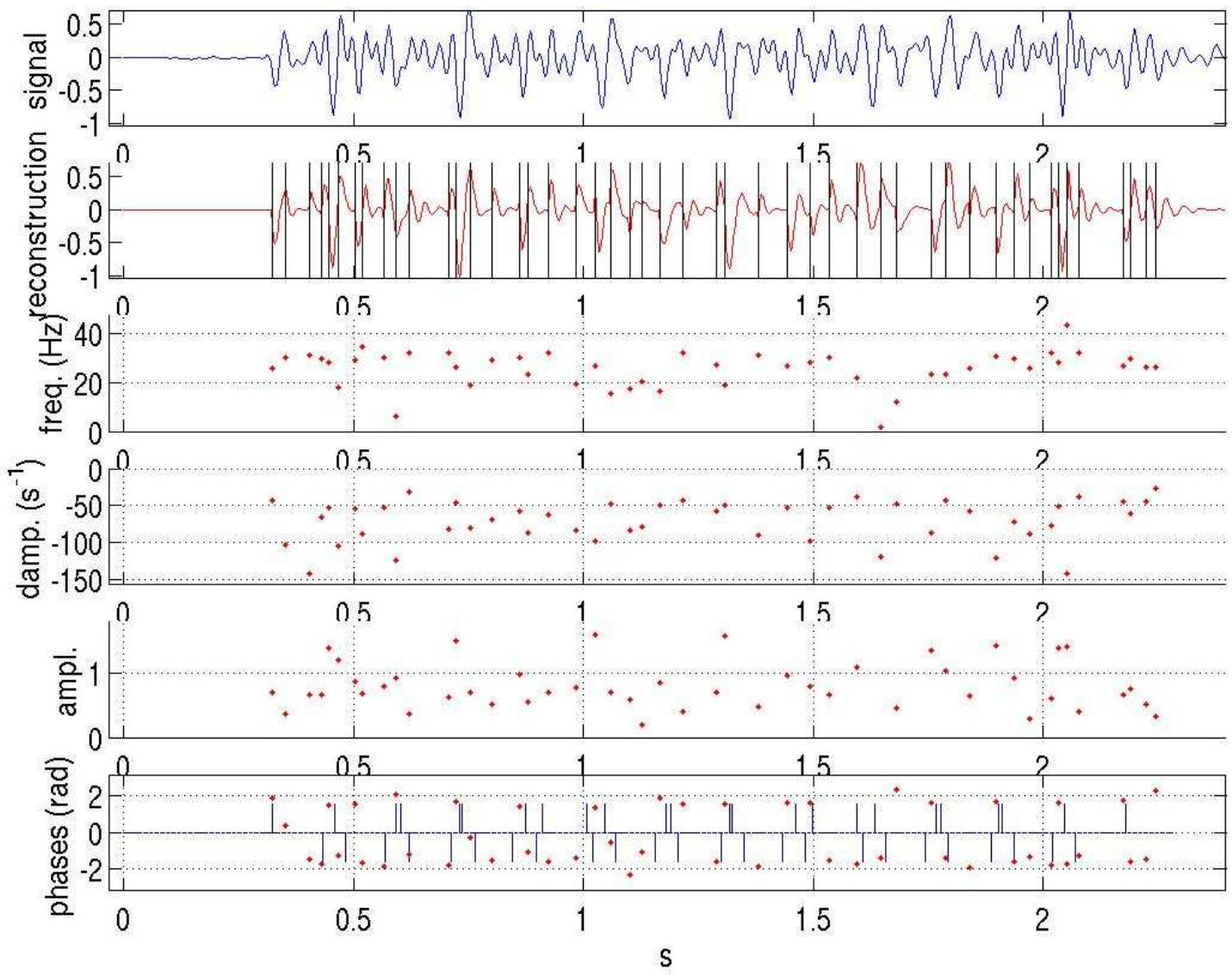

Fig. 16. Time-frequency Prony method applied to a recorded signal at speed $4.16 \mathrm{~m} / \mathrm{s}$. From top to bottom: Real signal after lowpass filtering; Detected shock instants (vertical lines) and signal reconstruction by time-frequency Prony $\left(P=2, N_{f}=10\right) ; E$ stimated frequencies versus time; Estimated damping factors versus time, Estimated amplitudes versus time; Estimated phases versus time. On last curve, the vertical lines indicate the shock instants of the excitation model.

\section{References}

[ATA 82] Atal B. S., RemdeJ. M., A new model of LPC excitation for producing natural speech at low bite rates, Proceedings of ICASSP 1982, pp. 614, 618.

[BAS 68] Bass J., Cours de mathématiques, Tome II, Editeurs Masson et Cie, 1968.

[BLA 75] Van Blaricum M. L., Mittra R., A technique for extracting the poles and residues of a system directly from its transient response, IEEE Trans. Antennas Propagat., Vol. AP-23, pp. 777-781, Nov. 1975.

[BRI 81] Brillinger D.R., Times Series data analysis and theory, Holden-Day, 1981.

[BRE 86] Bresler Y., Macowski A., Exact maximum likelihood parameter estimation of superimposed exponential signals in noise, IEEE Trans. On Acoust., Speech, Signal processing, Vol. ASSP-34, pp. 1081-1089, Oct. 1986.

[BRU 55] Bruhat G., Cours de Physique Générale, Mécanique, Chapitre XVII, 5ème édition, Editeur MASSON, 1955.

[CAS 01] Castanié F., Analyse spectrale, Traité IC2, Editeur HERMES, 2002.

[CEC 87] C.E.C.M. (Convention Européenne de la Construction Métallique), Recommandations pour la vérification à la fatigue des structures en acier, Construction métallique, $\mathrm{N}^{\circ} 1$, Mars 1987, 24ème année, pp. 3, 33.

[COM 02] Combet F, Martin N., Jaussaud P., Recovery of a high shock probability process using blind deconvolution methods, EUropean SIgnal Processing COnference, EUSIPCO 20002, Toulouse, France, Vol. II, pp. 91-94, September 3-6, 2002. 
[DON 68] McDonough R. N., Huggins W. H., Best least-squares representation of signals by exponentials, IEEE Trans. Automatic. Contr., Vol. AC-13, pp. 408-412, Aug. 1968.

[DUR 99] Durnerin M., Une stratégie pour l'interprétation en analyse spectrale, détection et caractérisation des composantes d'un spectre, PhD thesis, INPG, Sept. 211999.

[GAR 00] Garoosi V., Jansen B.H., Development and evaluation of the piecewise Prony method for evoked potentiel analysis, IEEE Trans. On Biomedical Engineering, Vol. 47, º12, pp. 1549-1554, Dec. 2000.

[GOL 83] Golub G.H., Van Loan C. F., Matrix computations, The Johns Hopkins University Press, 1983.

[HEN 81] Henderson T.L., Geometric methods for determining system poles from transient response, IEEE Trans. On Acoust., Speech, Signal Processing, Vol. ASSP-29, pp. 982-988, Oct. 1981.

[HOR 02] Hory C., Martin N., "Maximum Likelihood noise estimation for segmentation spectrogram control”, ICASSP 02, Orlando, Florida, Vol. II pp. 1581-1584, May 13-17, 2002.

[HUN 72] Hunt B. R., A theorem on the difficulty of numerical deconvolution, IEEE Trans. on Audio and Electroacoustics, Vol. 20, Mars 72, pp. 94-95.

[KUM 82] Kumaresan R., Tuft D. W., Estimating the parameters of exponentially damped sinusoids and pole-zero modeling in noise, IEEE Trans. On Acoust., Speech, Signal Processing, Vol. ASSP-30, pp. 83-840, Dec. 1982.

[KUM 83] Kumaresan R., On the zeros of the linear prediction-error filter for deterministic signals, IEEE Trans. On Acoust., Speech, Signal Processing, Vol. ASSP-31, pp. 637-640, Feb. 1983.

[KUM 86] Kumaresan R., Scharf L. L., Shaw A. K., An algorithm for pole-zero modeling and spectral analysis, IEEE Trans. On Acoust., Speech, Signal Processing, Vol. ASSP-34, pp. 217-220, June 1986.

[LAC 97] Lacoume J. L., Amblard P. O., Comon P., Statistiques d'ordre supérieur pour le traitement du signal, Masson, 1997.

[LAL 99] Lalanne C., Vibrations et chocs mécaniques, Vibrations sinusoïdales, Tome 1, Hermès, 1999.

[LEE 98] Lee J.Y., Nandi A.K., Blind deconvolution of impacting signals using higher-order statistics, MSSP, Vol.12 (2), pp.357-371, 1998.

[MAR 87] Marple S. L. Jr., Digital Spectral Analysis with Applications, Englewood Cliffs, NJ, Prentice Hall, 1987.

[MAR 02] Martin N, Durnerin M, Silvent A., Co-auteurs: Aquilina R., Castanié F., Goullet G., Lacoume J.-L., Lejeune G., Mailhes C., d'Urso G., ASPECT: Une stratégie pour l'interprétation en analyse spectrale, to published, EYROLLES Editor, EDF Collection.

[NEH 82] Nehorai A., Morf M., Enhancement of sinusoids in colored noise and the whitening performance of exact least-squares predictors, IEEE Trans. On Acoust., Speech, Signal Processing, Vol. ASSP-30, pp. 353-363, June 1983.

[NEW 89] Newland D.E., Mechanical Vibration Analysis and Computation, Longman, Singapore, 1989.

[PAP 90] Papadopoulos C. K., Nikias C. L., Parameter estimation of exponentially damped sinusoids using higher order statistics, IEEE Trans. On Acoust., Speech, Signal Processing, Vol. ASSP-38, pp. 1424-1435, Aug. 1990.

[PRO 1795] Prony R., Essai expérimental et analytique, Sur les lois de la dilatabilité des fluides élastiques et sur celles de la force expansive de la vapeur de l'eau et de la vapeur de l'alkool, à différentes températures, Journal de l'Ecole Polytechnique, Paris 1, pp. 24-76, 1795.

[ROB 96] Robert T., Modélisation continue de signaux non-stationnaires à rupture brutale, PhD Thesis, INP Toulouse, January 15, 1996.

[SAK 86] Sakaï H., Estimation of frequencies of sinusoids in colored noise, Proceedings of ICASSP 86, Japan, Tokyo, pp. 177-180, 1986.

[SAN 99] Sanchez-Gasca J. J., Chow J. H., Performance comparison of three identification methods for the analysis of electromechanical oscillations, IEEE Trans. On Power Systems, Vol. 14, N³, August 1999.

[SAT 78] Satorius E.H., Zeidler J. R., Maximum entropy spectral analysis of multiple sinusoids in noise, Geophysics, Vol. 43, pp. 1111-1118, Oct. 1978.

[SHA 94] Shaw A. K., A decoupled approach for optimal estimation of transfer function parameters from input-output data, IEEE Trans. on Signal Processing, Vol. 42, pp. 1275-1278, May 1994.

[SHA 01] Shaw A. K., Naishadham K., ARMA-based time-signature estimator for analysing resonant structures by the FDTD method, IEEE Trans. on Antennas and Propagation, Vol. 49, N³, pp. 327-329, March 2001.

[STE 65] Steiglitz K., McBride L. E., A technique for the identification of linear systems, IEEE Trans. Automatic Control, Vol. AC-10, pp. 461-464, Octobre 1965. 
[STO 81] Stoïca P., Söderström T., The Steiglitz-McBride identification algorithm revisited - Convergence analysis and accuracy aspects, IEEE Trans. Automatic Control, Vol. AC-26, N³, pp. 712-717, June 1981.

[TAL 01] Taleb A., Sole J., Jutten C., Quasi-Nonparametric Blind inversion of wiener systems, IEEE Trans. on signal Processing, Vol. 49, N5, May 2001, pp. 917- 924.

[YOU 01] Younan N. H., LEE H. S., Mazzola S., Estimating the model parameters of deep-level transient spectroscopy data using a combined wavelet/singular value decomposition Prony method, Review of Scientific Instruments, Vol. 72 , №3, pp. 1800-1805, March 2001

[YVE 93] Yvetot S., Mailhes C., Biotteau J. C., L'analyse de Prony multi-modèle et multi-date de signaux transitoires, GRETSI Juan-Les-Pins, France, September, 13-16, 1993.

[YVE 96] Yvetot S., Analyse de Prony multi-modèle de signaux transitoires, PhD Thesis, INP Toulouse, November 15, 1996.

\section{Appendix A: Exact Prony solution}

When $\mathrm{N}$, the number of points in the signal $y[k]$, equals $2 \mathrm{P}$ we have as many equations of type Eq. (18) as parameters to determine. The system thus has an exact solution $y[k]=\hat{y}[k]$ and Eq. (21) becomes

$$
\sum_{p=0}^{P} \eta_{p} y[k-p]=0 \quad \forall k=P, N \text { and } N=2 P
$$

This equation leads to parameters $\eta_{p}$ from which we can deduce poles $z_{p}$ by polynomial factorization of the denominator of Eq. (19)

$$
\prod_{p=1}^{P}\left(1-z_{p} z^{-1}\right)=\sum_{p=0}^{P} \eta_{p} z_{p}^{-1}
$$

In the second step we have to determine $a_{p}$, which can be done by using Eq. (18) after inverting the obtained matrix.

\section{Appendix B: Least-squares Prony solution}

When $N>2 P$, we have more equations than parameters to find. The solution of this system can only be approached. Thus an error exists. This error, between the signal and the model $\hat{y}[k]$, we denoted by $\varepsilon[k]$. Then $y[k]=\hat{y}[k]+\varepsilon[k]$. Eq. (21) becomes

$$
\sum_{p=0}^{P} \eta_{p} y[k-p]=\sum_{p=0}^{P} \eta_{p} \varepsilon[k-p] \forall k=P, N \text { and } N>2 P
$$

This turns out to be an ARMA $(\mathrm{P}, \mathrm{P})$ model whose $P$ MA coefficients are identical to $P$ AR coefficients. Thus the first step is to solve Eq. (B.1) for coefficients $\eta_{p}$ by minimizing the error power

$$
\eta_{p} / \sum_{k=P}^{N}|\varepsilon[k]|^{2} \text { minimum }
$$

Exactly as for the exact model, poles $z_{p}$ can be deduced from Eq. (A.2) and amplitudes $a_{p}$ are calculated from Eq. (18) using a least-squares method. Error $\varepsilon[k]$ also contains the noise included in the signal, if any. The least-squares Prony method - or extended Prony - more often introduces an extra approximation when calculating coefficients $\eta_{p}$ from an AR model. An AR model of signal $y[k]$ can be written as

$$
\sum_{p=0}^{P} \eta_{p} y[k-p]=e_{l s}[k] \quad \forall k=P, N \text { and } N>2 P
$$

with $e_{l s}[k]$ the prediction error power. Coefficients $\eta_{p}$ can be found by minimizing the error power when predicting $e_{l s}[k]$ instead of $\varepsilon[k]$. Such an approach is equivalent to whitening the error $e_{l s}[k]$ in Eq. (B.3) whereas this noise theoretically results from an MA process, see Eq. (B.1), which is not white of course. 


\section{Appendix C: Resolution of the two steps correlation Prony}

In the first step, the least-squares solution of Eq. (23) is written $\hat{\boldsymbol{\eta}}$. Then

$$
\hat{\boldsymbol{\eta}}=-\Gamma^{\#} \gamma
$$

where $\boldsymbol{\Gamma}^{\#}=\left(\begin{array}{ll}\boldsymbol{\Gamma}^{H} & \boldsymbol{\Gamma}\end{array}\right)^{-1} \boldsymbol{\Gamma}^{H}$ is the pseudo-inverse matrix of $\boldsymbol{\Gamma}$. According to Eq. (18) the poles $z_{p}$, roots of the characteristic function (A.2) give the frequencies and damping factors for each mode $(p)$

$$
\left\{\begin{array}{l}
\alpha_{p}=-\ln \left(\left|z_{p}\right|\right) / t_{e} \\
f_{p}=\operatorname{angle}\left(z_{p}\right) / 2 \pi t_{e}
\end{array}\right.
$$

For a real signal, the AR coefficients are real. Thus, the poles are either real (this corresponds to non oscillating solutions) or complex conjugate (oscillating solution).

In the second step, when the poles $z_{p}$ are substituted in Eq. (18), the system is now linear $\mathbf{Z} \mathbf{a}=\left(\begin{array}{ccc}1 & \ldots & 1 \\ z_{1} & \ldots & z_{P} \\ \ldots & \ldots & \ldots \\ z_{1}^{N-1} & \ldots & z_{P}^{N-1}\end{array}\right)\left(\begin{array}{c}a_{1} \\ a_{2} \\ \ldots \\ a_{P}\end{array}\right)=\left(\begin{array}{c}y[1] \\ y[2] \\ \ldots \\ y[N]\end{array}\right)=\mathbf{y}$

where $\mathbf{Z}$ is a $N \times P$ Vandermonde matrix. The least-squares solution of Eq. (C.3) which we denote $\hat{\mathbf{a}}$, can be written as follows

$$
\hat{\mathbf{a}}=\mathbf{Z}^{\#} \quad \mathbf{y}
$$

where $\mathbf{Z}^{\#}=\left(\begin{array}{lll}\mathbf{Z}^{H} & \mathbf{Z}\end{array}\right)^{-1} \quad \mathbf{Z}^{H}$ is the pseudo-inverse of $\mathbf{Z}$. Using Eq. (18) we get

$$
\left\{\begin{array}{l}
A_{p}=\left|a_{p}\right| \\
\Phi_{p}=\operatorname{angle}\left(a_{p}\right)
\end{array}\right.
$$

The amplitudes are real or complex conjugate, so that the mode linear combinations give real functions. 